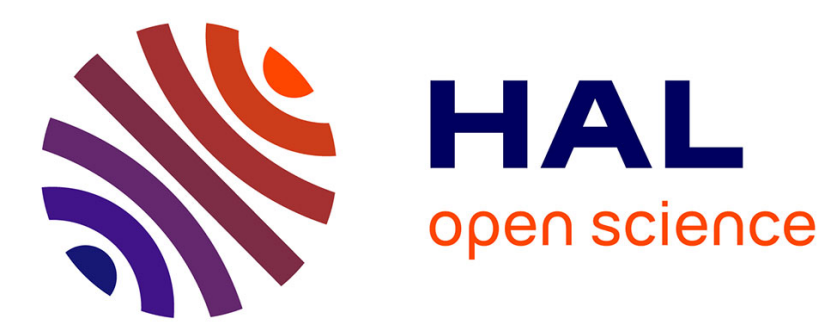

\title{
Atomes "habillés" par des photons optiques ou de radiofréquence \\ C. Cohen-Tannoudji
}

\section{To cite this version:}

C. Cohen-Tannoudji. Atomes "habillés" par des photons optiques ou de radiofréquence. Journal de Physique Colloques, 1971, 32 (C5), pp.C5a-11-C5a-28. 10.1051/jphyscol:1971502 . jpa-00214665

\section{HAL Id: jpa-00214665 https://hal.science/jpa-00214665}

Submitted on 1 Jan 1971

HAL is a multi-disciplinary open access archive for the deposit and dissemination of scientific research documents, whether they are published or not. The documents may come from teaching and research institutions in France or abroad, or from public or private research centers.
L'archive ouverte pluridisciplinaire HAL, est destinée au dépôt et à la diffusion de documents scientifiques de niveau recherche, publiés ou non, émanant des établissements d'enseignement et de recherche français ou étrangers, des laboratoires publics ou privés. 


\title{
ATOMES « HABILLÉS » PAR DES PHOTONS OPTIQUES OU DE RADIOFRÉQUENCE
}

\author{
C. COHEN-TANNOUDJI
}

Laboratoire de Spectroscopie Hertzienne de l'E. N. S. Paris

\begin{abstract}
Résumé. - Un atome irradié par des photons non résonnants peut les absorber et réémettre virtuellement; il est en quelque sorte " habillé ) par les photons du champ électromagnétique dans lequel il est plongé. Nous montrons dans cet article comment les propriétés physiques d'un atome " habillé » par des photons optiques ou de radiofréquence diffèrent de celles de l'atome isolé et décrivons les expériences qui ont permis de mettre en évidence les effets correspondants. Quelques applications pratiques sont également envisagées.
\end{abstract}

Abstract. - An atom irradiated by non resonant photons can absorb and reemit them virtually ; it may be considered as (cdressed) by the photons of the surrounding electromagnetic field. We show in this article how the physical properties of atoms dressed by optical and $R F$ quanta differ from the ones of isolated atoms and we describe the experimental study of these effects. Some practical applications are also considered.

Avant de commencer cet exposé, je voudrais dire à quel point je suis touché par la marque d'estime et de confiance qui m'est témoignée par la Société Française de Physique avec l'attribution de ce grand prix de Physique Jean Ricard; à quel point aussi je suis impressionné d'avoir été ainsi choisi pour la première fois, et parmi tant de physiciens si compétents.

Je ne saurais également exprimer assez toute la reconnaissance que j'éprouve à l'égard des deux personnes qui m'ont appris à découvrir et à aimer la physique, je veux parler de MM. Kastler et Brossel. C'est pour moi, je crois, une chance exceptionnelle que d'avoir pu, à leur contact, acquérir ma formation de chercheur.

Les travaux dont je vais vous parler tout à l'heure ont été réalisés au Laboratoire de Physique de l'Ecole Normale Supérieure, au sein d'une équipe de jeunes chercheurs enthousiastes, avec qui j'ai été très heureux de pouvoir travailler. Il s'agit entre autres de Nicole Polonsky-Ostrowsky, Serge Haroche, Jacques DupontRoc, Franck Laloë, Claire Landré, Maryvonne Le Dourneuf, Gilbert Grynberg et bien d'autres. Je voudrais les associer pleinement à l'hommage qui m'est rendu aujourd'hui, de même que tous les autres chercheurs, techniciens et administratifs du Laboratoire dont l'aide nous a été si précieuse.

Nous tous qui sommes réunis à ces journées de physique d'Evian savons les efforts qui ont été déployés par les responsables actuels de la Société Française de Physique et ceux qui les ont précédés au cours des dernières années pour donner à notre Société un visage jeune, attrayant et dynamique. A cet égard, le geste de $M$. et $M^{\text {me }}$ Ricard aura cerfainement, par sa noblesse, contribué à rehausser de façon éclatante le prestige de notre Société et celui de la recherche fondamentale. Je crois traduire l'opinion unanime des physiciens en leur adressant ici tous nos remerciements.

Introduction. - Lorsqu'on irradie un atome avec des photons dont la fréquence $v$ coïncide avec l'une des fréquences de Bohr atomiques $\left(E_{2}-E_{1} / h\right)$, il se produit en général une absorption résonnante d'un photon avec passage de l'atome du niveau inférieur $\left|E_{1}\right\rangle$ au niveau supérieur $\left|E_{2}\right\rangle$. Si le niveau $\left|E_{1}\right\rangle$ est le niveau fondamental, le niveau $\mid E_{2}>$ le niveau excité de résonance et si les photons incidents sont polarisés, on sait qu'il apparaît des différences de population entre les divers sous-niveaux Zeeman de $\mid E_{2}>[1]$. La retombée de l'atome dans l'état fondamental par émission spontanée d'un photon fait apparaître également des différences de population entre les sous-niveaux Zeeman de $\left|\mathrm{E}_{1}\right\rangle$. C'est le principe bien connu du pompage optique [2] qui permet de transférer aux atomes une partie du moment cinétique transporté par les photons polarisés. L'inégalité de populations ainsi réalisée entre les sousniveaux Zeeman de $\mid E_{2}>$ ou $\mid E_{1}>$ peut être ensuite modifiée par des absorptions résonnantes de photons de radiofréquence ; la mesure précise de ces fréquences de résonance fournit des informations précieuses sur la structure interne des niveaux $\left|E_{2}\right\rangle$ et $\left|E_{1}\right\rangle$.

Les considérations précédentes pourraient faire croire que les effets de l'interaction entre l'atome et les photons incidents ne se manifestent qu'à résonance, c'est-à-dire lorsque l'énergie globale est conservée à l'issue du processus d'absorption. En fait, il n'en est rien et le but de cet exposé est de décrire quelques effets liés au couplage entre un atome et des photons 
optiques ou de radiofréquence non résonnants. La conservation de l'énergie est en effet moins stricte en mécanique quantique qu'en mécanique classique, et le système global atome + champ électromagnétique peut passer intermédiairement par des états dont l'énergie est différente de celle de l'état initial et final, pourvu que la durée de cette transition intermédiaire, appelée encore transition virtuelle, soit suffisamment courte (inférieure à $\hbar / \Delta E$, où $\Delta E$ est le défaut d'énergie). L'atome peut donc absorber et réémettre virtuellement les photons non résonnants qui arrivent sur lui; pour visualiser ce processus, nous dirons que l'atome est « habillé » par les photons du champ dans lequel il est plongé. Comme nous allons le voir, sés propriétés physiques ne sont plus les mêmes que celles de l'atome isolé.

Les effets que nous allons étudier peuvent être qualifiés de «stimulés » dans la mesure où ils sont provoqués par des photons incidents. Il existe également des effets «spontanés» bien connus qui apparaissent en l'absence de tout photon incident et qui jouent un rôle fondaméntal en électrodynamique quantique [3]. C'est ainsi qu'un atome ou un électron, isolé peut émettre spontanément un photon et le réabsorber virtuellement. On peut montrer qu'il en résulte entre autres effets un déplacement des niveaux atomiques appelé «Lamb-shift » et une anomalie du moment magnétique de spin de l'électron libre par rapport à la valeur prévue par la théorie de Dirac (anomalie g-2). Nous tâcherons au cours de cet exposé d'établir un parallèle étroit entre ces effets spontanés et les effets stimulés qui apparaissent lors d'une irradiation non résonnante.

A. Interaction d'un atome avec des photons optiques non résonnants. - Dans cette première partie, nous nous proposons d'étudier l'effet d'une irradiation lumineuse non résonnante sur l'état fondamental d'un atome. Auparavant, rappelons quelques propriétés bien connues de l'émission spontanée.

1. RAPPELS SUR L'ÉMISSION SPONTANÉE. - Un atome est initialement dans le niveau excité $\mid \mathrm{e}>$ d'énergie $E_{0}$ au-dessus du fondamental (Fig. 1a). L'interaction avec le champ électromagnétique couple cet état à toute une série d'autres états où l'atome se trouve dans le niveau fondamental $\mid \mathbf{f}>$ en présence d'un photon d'énergie $E$ (Fig. $1 b$ ). La résolution de l'équation de Schrödinger du système permet de montrer que, sous l'effet de ce couplage, l'énergie $E_{0}$ de l'état initial doit être remplacée par

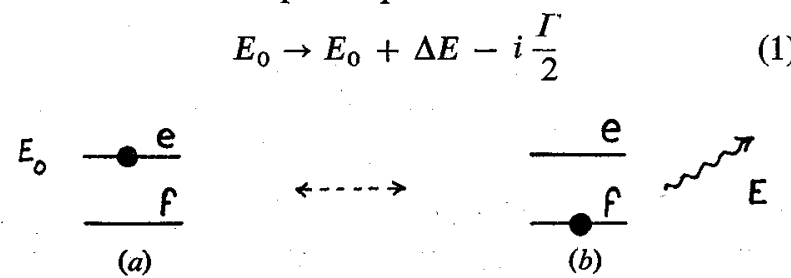

FIG. 1. - Etats du système intervenant dans le processus d'émission spontanée. où $\Delta E$ et $\Gamma$ sont des constantes réelles [4]. Le facteur d'évolution temporelle en $\mathrm{e}^{-i E_{0} t}$ (nous prenons $\hbar=1$ devient par suite

$\exp \left(-i E_{0} t\right) \rightarrow \exp \left(-i\left(E_{0}+\Delta E\right) t\right) \exp \left(-\Gamma \frac{t}{2}\right)$

et la probabilité de trouver l'atome dans l'état excité décroît exponentiellement en $\mathrm{e}^{-\Gamma t}$. Cette évolution irréversible de l'état excité est due à la possibilité pour l'atome d'émettre réellement un photon (Fig. 2a)

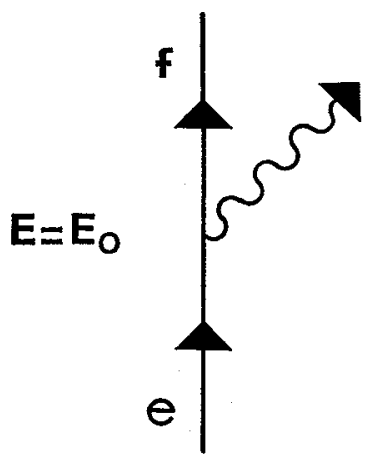

(a)

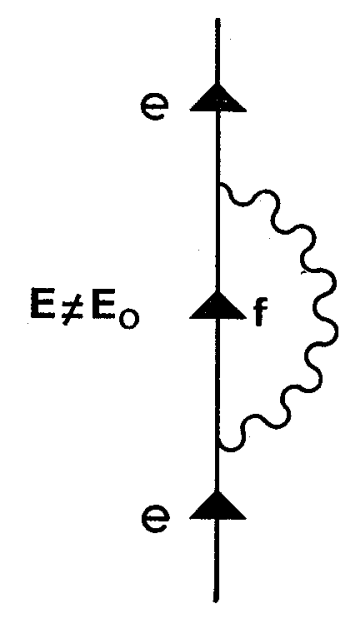

(b)

FIG. 2.

$2 a$. - Emission réelle d'un photon.

$2 b$. - Emission et réabsorption virtuelle d'un photon.

avec conservation de l'énergie globale : $E=E_{0}$. Si $E \neq E_{0}$, le couplage entre les états des figures $1 a$ et $1 b$ continue cependant d'exister et peut être décrit en termes d'émissions et de réabsorptions virtuelles d'un photon par 1'atome (Fig. 2b). Ce sont ces processus qui sont responsables du déplacement en énergie $\Delta E$ de l'état excité (Lamb-shift).

2. ETUDE DU PROCESSUS D'ABSORPTION. - L'état initial correspond maintenant à l'atome dàns le niveau $|\mathrm{f}\rangle$ en présence d'un photon incident d'énergie $E_{i}$ (Fig. 3a). Cet état est couplé aux états des figures $3 b$ puis $3 c$, d'énergie $E_{0}$ et $E$ et déjà décrits plus haut. 


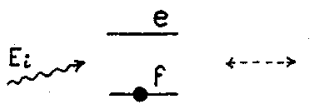

(a)

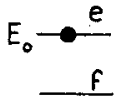

(b)

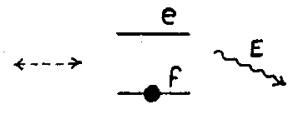

(c)
Fig. 3. - Etats du système intervenant dans le processus d'absorption.

Pour déterminer l'évolution du système, on peut prendre le point de vue simple suivant : dans le domaine optique, l'absorption est faible devant l'émission spontanée; le couplage entre l'état $3 b$ et l'état $3 a$ comprenant un photon unique est faible devant le couplage entre l'état $3 b$ et l'ensemble des états $3 c$ (l'énergie $E$ et la direction du photon émis peuvent être quelconques). L'évolution de l'état $3 b$ est donc essentiellement déterminée par le couplage avec $3 c$ et peut être décrite, comme nous l'avons vu plus haut, en remplaçant $E_{0}$ par $\widetilde{E}_{0}-i \Gamma / 2\left(\right.$ avec $\widetilde{E}_{0}=E_{0}+\Delta E$ ). On est alors ramené à un problème plus simple où l'état initial d'énergie $E_{i}$ est couplé à un seul état dont l'énergie est complexe $\left(\widetilde{E}_{0}-i \Gamma / 2\right)$, c'est-à-dire possédant une certaine largeur (Fig. 4).

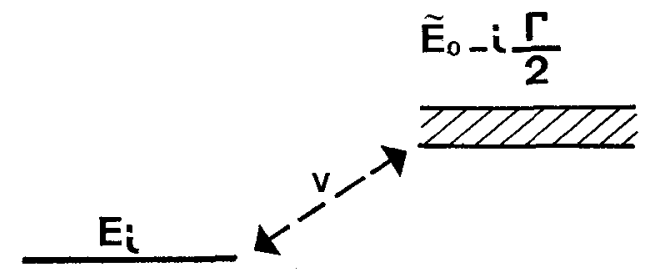

$|v| \ll \Gamma$

FIG. 4. - Modèle simple pour l'étude du processus d'absorption.

Si $v$ désigne ce couplage, il s'agit de diagonaliser la matrice

$$
\left(\begin{array}{cc}
E_{i} & v \\
v^{*} & \widetilde{E}_{0}-i \Gamma / 2
\end{array}\right)
$$

avec la condition

$$
|v| \ll \Gamma
$$

puisque l'absorption est faible devant l'émission spontanée.

La matrice (3) étant non hermitique, celle de ses valeurs propres qui tend vers $E_{i}$ lorsque $|v|$ tend vers zéro est en général complexe. L'effet du couplage est donc de remplacer $E_{i}$ par

$$
E_{i} \rightarrow E_{i}+\Delta^{\prime}-i \frac{\Gamma^{\prime}}{2}
$$

où $\Delta^{\prime}$ et $\Gamma^{\prime}$ sont des quantités réelles que l'on peut interpréter comme représentant respectivement le déplacement et l'instabilité (ou encore l'élargissement) de l'état fondamental dus à l'interaction avec le photon incident. 2 cas limites sont particulièrement intéressants à étudier.

i) $E_{i}=\widetilde{E}_{0}$ le photon incident est en résonance avec l'énergie $\widetilde{E}_{0}$ de la transition atomique (énergie $E_{0}$ corrigée du Lamb-shift $\Delta E$ ). On trouve alors aisément que :

$$
\left\{\begin{array}{l}
\Delta^{\prime}=0 \\
\Gamma^{\prime}=\Gamma\left(\frac{|v|}{\Gamma}\right)^{2} .
\end{array}\right.
$$

Le déplacement de l'état fondamental est nul. Par contre, l'instabilité de l'état excité est partiellement communiquée à l'état fondamental. (Notons que si $\Gamma$ était nul, le couplage entre les 2 états non perturbés de même énergie aurait eu au contraire pour effet de repousser les 2 états d'une quantité proportionnelle à $|v|$, sans leur conférer aucune instabilité. C'est d'ailleurs ce que l'on trouverait à partir de (3), si l'on prenait la limite $|v| \gg \Gamma$, opposée de (4).)

ii) $\left|E_{i}-\widetilde{E}_{0}\right| \gg \Gamma$. Le photon incident est non résonnant puisque son énergie diffère de celle de la transition atomique d'une quantité supérieure à la largeur naturelle du niveau excité. On trouve alors que

$$
\left\{\begin{array}{l}
\Delta^{\prime}=\frac{|v|^{2}}{E_{i}-\widetilde{E}_{0}} \\
\Gamma^{\prime}=\Gamma\left(\frac{|v|}{E_{i}-\widetilde{E}_{0}}\right)^{2} .
\end{array}\right.
$$

Dans ce cas, l'état fondamental est déplacé d'une quantité supérieure à son élargissement puisque

$$
\left|\frac{\Delta^{\prime}}{\Gamma^{\prime}}\right|=\frac{\left|E_{i}-\widetilde{E}_{0}\right|}{\Gamma} \gg 1 .
$$

On voit d'autre part sur (7a) que le signe de $\Delta^{\prime}$ est le même que celui de $E_{i}-\widetilde{E}_{0}$.

Tous les résultats précédents demeurent valables dans un traitement plus rigoureux [5], [6] du problème où l'on résout l'équation de Schrödinger décrivant l'évolution du vecteur d'état développé sur les états de base des figures $3 a, 3 b, 3 c$, où l'on tient compte d'une structure Zeeman des niveaux $|\mathrm{f}\rangle$ et $|\mathrm{e}\rangle$, et où l'on suppose que plusieurs photons incidents arrivent sur l'atome avec une distribution donnée en énergie. La figure 5 représente les variations de $\Gamma^{\prime}$ et $\Delta^{\prime}$ avec $E_{\mathrm{i}}-\widetilde{E}_{0}\left(E_{i}\right.$ représente alors le centre de la raie excitatrice). La largeur des courbes correspondantes qui ont des formes d'absorption et de dispersion est de l'ordre de la largeur de la raie excitatrice. On retrouve bien que $\Delta^{\prime}$, qui est proportionnel à l'intensité lumineuse totale, est nul pour $E_{i}=\widetilde{E}_{0}$ alors que $\Gamma^{\prime}$ est maximum en ce point. Par contre, pour $\left|E_{i}-\widetilde{E}_{0}\right|$ suffisamment grand, $\Delta^{\prime}$ est plus grand que $\Gamma^{\prime}$ et a le même signe que $E_{i}-\widetilde{E}_{0}$. 


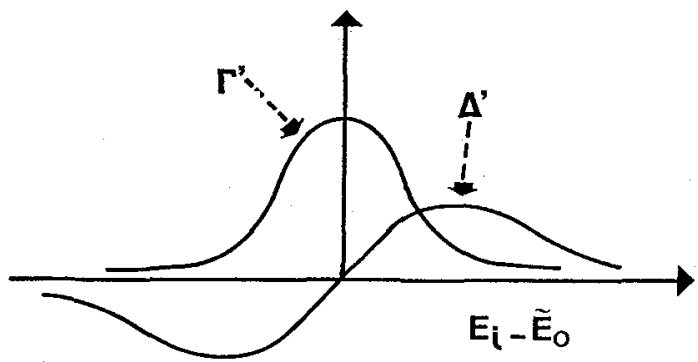

FIG. 5. - Variations de $\Delta^{\prime}$ et $\Gamma^{\prime}$ en fonction de l'écart à résonance $E_{i}-E_{0}$.

On voit également que ce sont les processus d'absorption réelle d'un photon incident $\left(E_{i}=\widetilde{E}_{0}\right)$ qui sont à l'origine de l'élargissement de l'état fondamental (Fig. 6a). Lorsque les photons incidents sont non résonnants $\left(E_{i} \neq \widetilde{E}_{0}\right)$, ils ne peuvent qu'être absorbés et réémis virtuellement (Fig. $6 b$ ). De tels processus

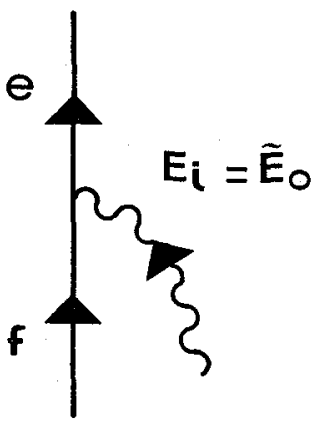

(a)

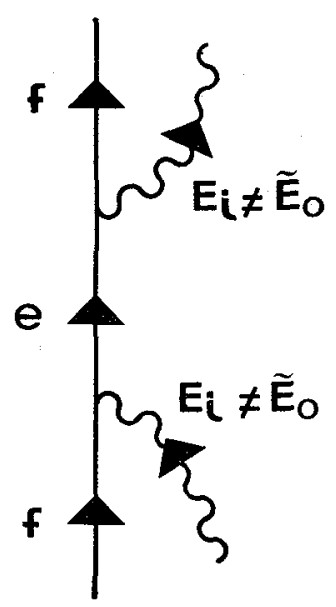

(b)

FIG. 6.

6a. - Absorption réelle d'un photon.

6b. - Absorption et réémission virtuelle d'un photon.

ont cependant une conséquence physique importante : ils déplacent le niveau atomique fondamental. Notons d'ailleurs que les conséquences de l'interaction atomephoton se manifestent également sur le photon par l'apparition dans l'indice de réfraction d'une partie réelle et d'une partie imaginaire décrivant respectivement les phénomènes de dispersion anormale et d'absorption. En particulier, on peut considérer que le photon ne se propage pas pendant qu'il est absorbé virtuellement par l'atome, ce qui explique la modification de sa vitesse de propagation.

Si les effets stimulés $\left(\Delta^{\prime}, \Gamma^{\prime}\right)$ associés au processus d'absorption peuvent être décrits au moyen des mêmes images que les effets spontanés $(\Delta, \Gamma)$ rappelés plus haut (comparer les figures $6 a$ et $6 b$ aux figures $2 a$ et $2 b$ ), ils présentent cependant avec ces derniers un certain nombre de différences.

Tout d'abord, comme $|v| \ll \Gamma$, l'ordre de grandeur de $\Delta^{\prime}$ et $\Gamma^{\prime}$ est, pour les sources lumineuses usuelles, beaucoup plus faible que celui de $\Gamma$ et $\Delta E$. En particulier, il est très difficile de détecter le déplacement $\Delta^{\prime}$ par une variation de la fréquence optique émise par l'atome puisque $\Delta^{\prime}$ est en général noyé dans la largeur naturelle $\Gamma$ du niveau excité, et a fortiori dans la largeur Doppler de la raie optique, la plupart $\mathrm{du}$ temps supérieure à $\Gamma\left({ }^{1}\right)$.

D'autre part, la symétrie des 2 types d'effets n'est pas la même. L'émission spontanée est un processus

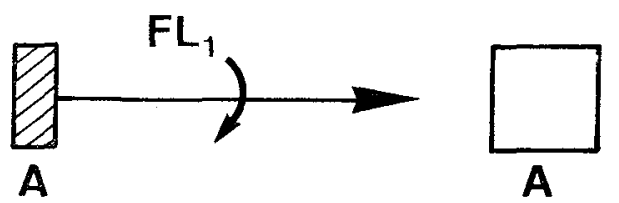

(a)

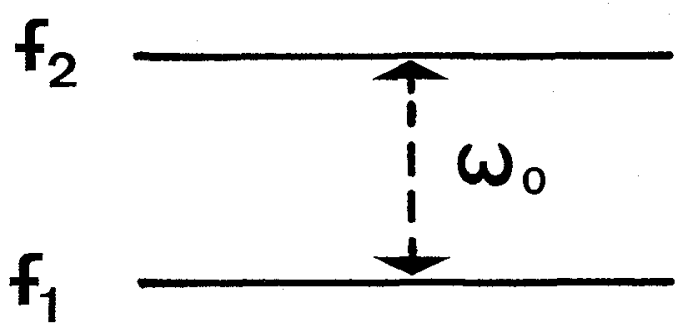

(b)

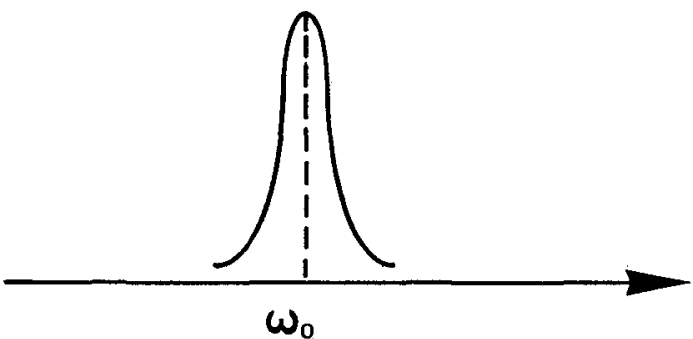

(c)

Fig. 7. - Mesure par des méthodes optiques d'une structure Zeeman dans l'état fondamental d'un atome. Schéma de principe.

(1) Signalons cependant que l'utilisation de sources laser très intenses a permis dans certains cas de détecter le déplacement $\boldsymbol{A}^{\prime}$ sur une raie optique [7]. 
isotrope : l'atome interagit avec tous les modes du champ électromagnétique associés à toutes les directions et polarisations possibles du photon émis. Il s'ensuit que les divers sous-niveaux Zeeman du niveau excité ont la même largeur naturelle et le même Lambshift. Dans le processus d'absorption par contre, l'atome interagit avec des photons de vecteur d'onde et de polarisation bien définis. Il en résulte que le déplacement et l'élargissement de l'état fondamental varient en général d'un sous-niveau Zeeman à l'autre.

Cette propriété importante est à la base des méthodes expérimentales d'étude du déplacement $\Delta^{\prime}$. Il est possible au moyen d'une expérience de pompage optique (Fig. $7 a$ ) de réaliser une différence de populations entre 2 sous-niveaux Zeeman $\left|f_{1}\right\rangle$ et $\left|f_{2}\right\rangle$ de l'état fondamental (Fig. 7b). Le même élément $\mathrm{A}$ est contenu dans la cellule de résonance et dans la source du faisceau lumineux de pompage $\mathrm{FL}_{1}$ qui est par suite résonnant. L'observation sur les signaux de détection optique de la raie de résonance magnétique (Fig. 7c) induite par un champ de radiofréquence résonnant entre $\left|f_{1}\right\rangle$ et $\left|f_{2}\right\rangle$ permet de déterminer avec une grande précision l'écart $\omega_{0}$ entre ces 2 sousniveaux. La perturbation introduite par $\mathrm{FL}_{1}$ se traduit essentiellement, par suite de son caractère résonnant, par un élargissement de la raie de résonance magnétique qui vient s'ajouter à la largeur intrinsèque de la raie due à la relaxation thermique (collision des atomes sur les parois de la cellule). Introduisons maintenant

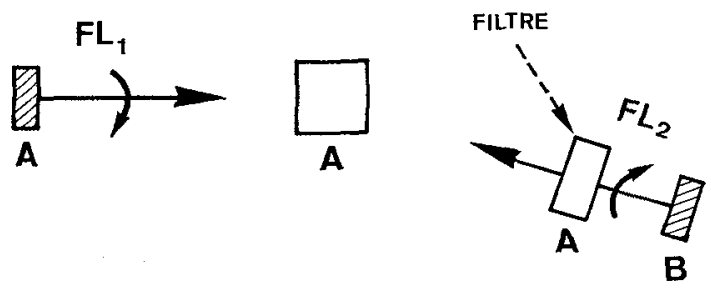

(a)

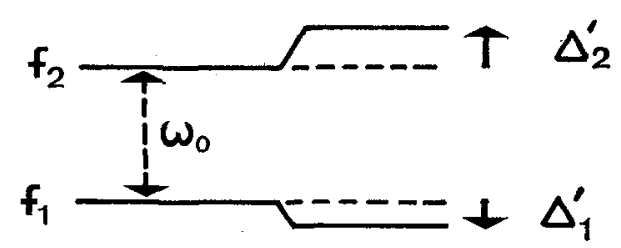

(b)

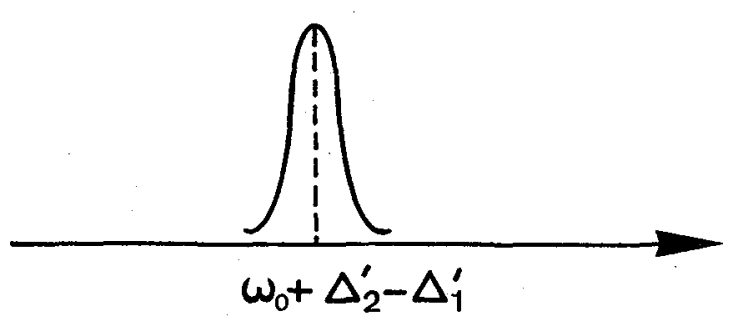

(c)

Fig. 8. - Mise en évidence ues déplacements énergétiques causés par une irradiation lumineuse non résonnante. Schéma de principe. un second faisceau lumineux $\mathrm{FL}_{2}$ dont la source contient un autre isotope B que celui contenu dans la cellule (Fig. 8a). Par suite du déplacement isotopique des raies optiques, l'irradiation due à $\mathrm{FL}_{2}$ est non résonnante. On choisit B de façon que l'écart entre $E_{i}$ et $\widetilde{E}_{0}$ ne soit pas trop loin de la valeur correspondant au maximum de $\Delta^{\prime}$ (cf. Fig. 5) $\left(^{2}\right.$ ). Un filtre rempli de l'élément $\mathrm{A}$ est interposé sur le trajet de $\mathrm{FL}_{2}$ et supprime les fréquences résonnantes contenues dans $\mathrm{FL}_{2}$. La perturbation due à $\mathrm{FL}_{2}$ se traduit donc essentiellement par un déplacement $\Delta^{\prime}$ qui, si la polarisation de $\mathrm{FL}_{2}$ est convenablement choisie, est différent pour $\left|f_{2}\right\rangle$ et $\left|f_{1}\right\rangle$ (Fig. $8 b$ ). Il en résulte que la raie de résonance magnétique détectée toujours au moyen du faisceau de pompage $\mathrm{FL}_{1}$ subit un déplacement $\Delta_{2}^{\prime}-\Delta_{1}^{\prime}$ (Fig. $8 c$ ) que l'on peut ainsi déterminer avec une grande précision.

Les premières expériences réalisées au moyen de cette méthode [6] avaient permis d'obtenir des déplacements des raies de résonance magnétique comparables à leur largeur et de vérifier en détail les prévisions théoriques concernant $\Delta^{\prime}$. Des améliorations successives apportées à l'expérience ont permis depuis d'augmenter considérablement le déplacement [8].

La figure 9 représente par exemple un résultat obtenu sur l'isotope ${ }^{199} \mathrm{Hg}$, de moment cinétique $I=\frac{1}{2}$ dans l'état fondamental. Le déplacement de la raie est près de 20 fois supérieur à sa largeur. On notera que le faisceau $\mathrm{FL}_{2}$ introduit un léger élargissement supplémentaire de la raie qui reste toutefois très inférieur au déplacement.

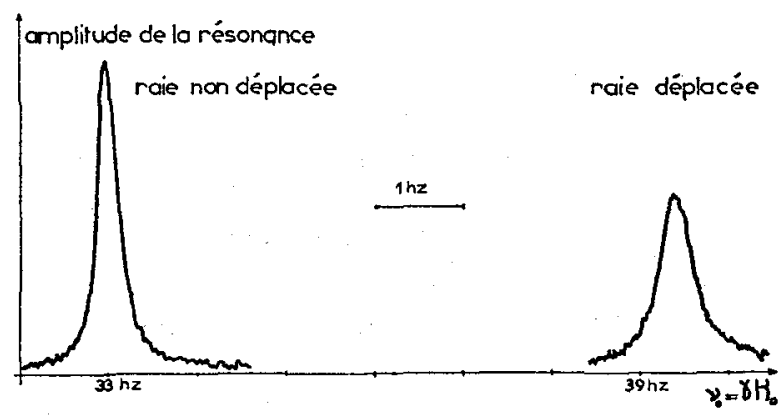

Fig. 9. - Résultat expérimental obtenu sur l'isotope ${ }^{199} \mathrm{Hg}$.

3. Champs Fictifs associés a UNE IRRADIATION LUMINEUSE NON RÉSONNANTE. - Nous avons vu plus haut toute l'importance du fait que le déplacement $\Delta^{\prime}$ varie d'un sous-niveau Zeeman à l'autre. Pour étudier ce point plus en détail, il faut introduire l'hamiltonien effectif $\mathfrak{H}_{\mathrm{e}}$ qui décrit l'effet de l'irradiation lumineuse non résonnante à l'intérieur de la multiplicité fondamentale.

(2) On peut également utiliser le déplacement Zeeman des raies optiques émises par une source contenant l'isotope A et placée dans un champ magnétique assez élevé. 
Si l'on suppose que le moment cinétique de l'état fondamental est $I=\frac{1}{2}$, la matrice représentant $\mathcal{H}_{\mathrm{e}}$ dans la base des 2 sous-niveaux Zeeman est hermitique et s'écrit

$$
\mathscr{H}_{\mathrm{e}}=\Delta^{\prime}\left(\begin{array}{ll}
\mathrm{A}_{11} & \mathrm{~A}_{12} \\
\mathrm{~A}_{12}^{*} & \mathrm{~A}_{22}
\end{array}\right) .
$$

Ses éléments, proportionnels à la quantité $\Delta^{\prime}$ définie plus haut ne dépendent autrement que du vecteur polarisation de la lumière incidente. Toute matrice hermitique $2 \times 2$, en particulier $\mathfrak{H}_{\mathrm{e}}$, peut être développée sur la matrice unité 1 et les 3 matrices de Pauli $\sigma_{x}, \sigma_{y}, \sigma_{z}$ :

$$
\mathscr{H}_{\mathrm{e}}=c_{0} 1+\sum_{i=x, y, z} c_{i} \sigma_{i} .
$$

Il s'ensuit que $\mathfrak{H}_{\mathrm{e}}$ peut être mise sous la forme d'une superposition linéaire des 3 composantes $I_{x}, I_{y}, I_{z}$ du moment cinétique $I$ et a par suite la même forme que celle d'un hamiltonien Zeeman correspondant à un champ magnétique statique $\mathbf{H}_{\mathrm{f}}$ dont les composantes seraient proportionnelles à $c_{x}, c_{y}, c_{z}$.

$$
\mathfrak{H}_{\mathrm{e}}=-\gamma \mathbf{I} \cdot \mathbf{H}_{\mathrm{f}}
$$

( $\gamma$ rapport gyromagnétique de l'état fondamental).

L'effet produit par une irradiation lumineuse non résonnante est donc identique à celui que l'on obtiendrait si l'on appliquait à l'atome un champ magnétique statique $\mathbf{H}_{\mathrm{f}}$ que nous qualifierons de «fictif 》 puisqu'il n'existe pas réellement $\left({ }^{3}\right)$. L'amplitude de ce champ est proportionnelle à $\Delta^{\prime}$, donc à l'intensité lumineuse totale. Sa direction peut s'obtenir à partir de simples arguments de symétrie. $\mathbf{H}_{\mathrm{f}}$ est invariant dans toute opération qui laisse le vecteur polarisation e de l'onde incidente inchangé. Si cette polarisation est circulaire droite, $\boldsymbol{H}_{\mathrm{f}}$ est nécessairement parallèle à la direction de propagation du faisceau lumineux.

L'équivalence entre l'effet de l'irradiation lumineuse non résonnante et celui d'un champ statique $\mathbf{H}_{\mathrm{f}}$ peut être démontrée expérimentalement de la façon suivante [9].

Des atomes ${ }^{199} \mathrm{Hg}$ sont placés en champ statique nul à l'intérieur d'un blindage magnétique qui les protège du champ terrestre (Fig. 10a). Ils sont pompés optiquement par un faisceau lumineux $\mathrm{FL}_{1}$, résonnant, qui se propage le long de $\mathrm{O} z$. Les spins atomiques sont donc orientés dans cette direction et ne subissent aucune précession de Larmor puisque le champ statique est nul. Un photomultiplicateur P. M. mesure la lumière transmise et fournit un signal dont on peut montrer aisément qu'il est proportionnel à $\left\langle I_{z}\right\rangle$, composante sur $\mathrm{O} z$ de l'orientation atomique $\langle\mathbf{I}\rangle$. Irradions alors brusquement les atomes ainsi orientés

(3) Le niveau fondamental est déplacé en plus globalement d'une quantíté $c_{0}$.

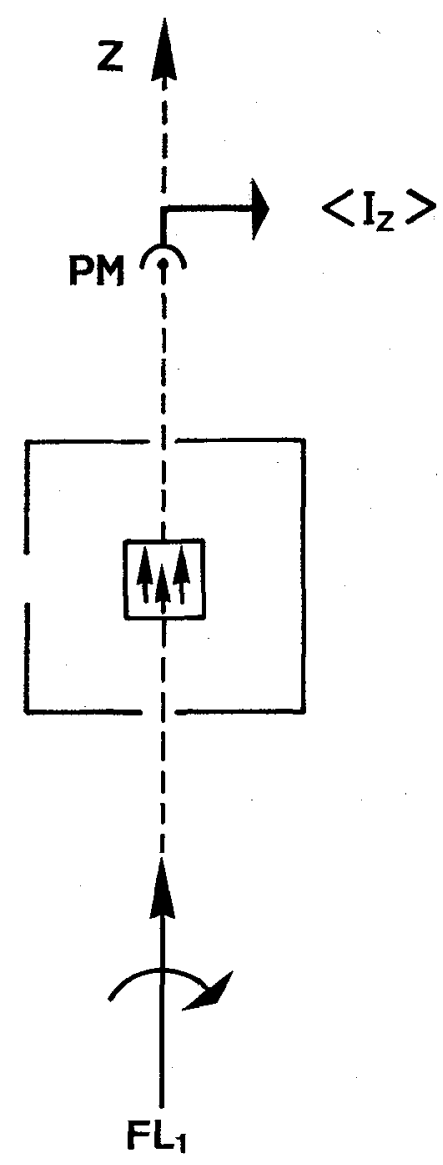

(a)

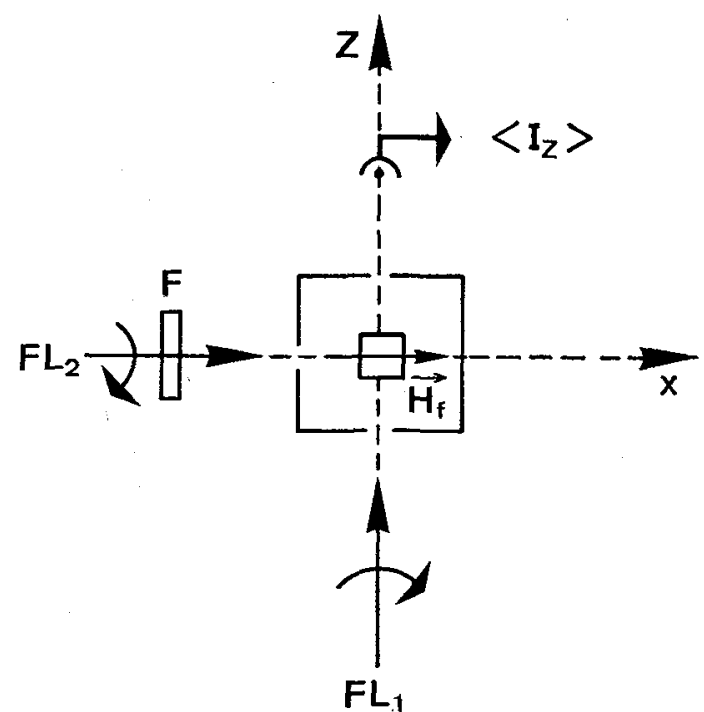

(b)

FIG. 10. - Mise en évidence du champ fictif $\mathbf{H}_{\mathrm{f}}$ associé à une irradiation lumineuse non résonnante. Schéma de principe.

par un faisceau lumineux non résonnant $\mathrm{FL}_{2}$ se propageant le long de $\mathrm{O} x$ et polarisé circulairement (Fig. 10b). Tout se passe comme si l'on appliquait brusquement un champ statique $\mathbf{H}_{\mathrm{f}}$ parallèlement à $\mathrm{O} x$. Les spins orientés le long de $\mathrm{O} z$, se mettent à 
précesser autour de $\mathrm{O} x$ avec une vitesse angulaire proportionnelle à $\left|\mathbf{H}_{\mathrm{f}}\right|$ et il en résulte une modulation de la composante $\left\langle I_{z}\right\rangle$ qui apparaît sur le signal fourni par P. M. La figure 11 montre un exemple d'une telle transitoire. Comme les déplacements des niveaux sont plus grands que leur largeur, on observe pendant le temps de relaxation plusieurs précessions de Larmor des spins autour du champ fictif.

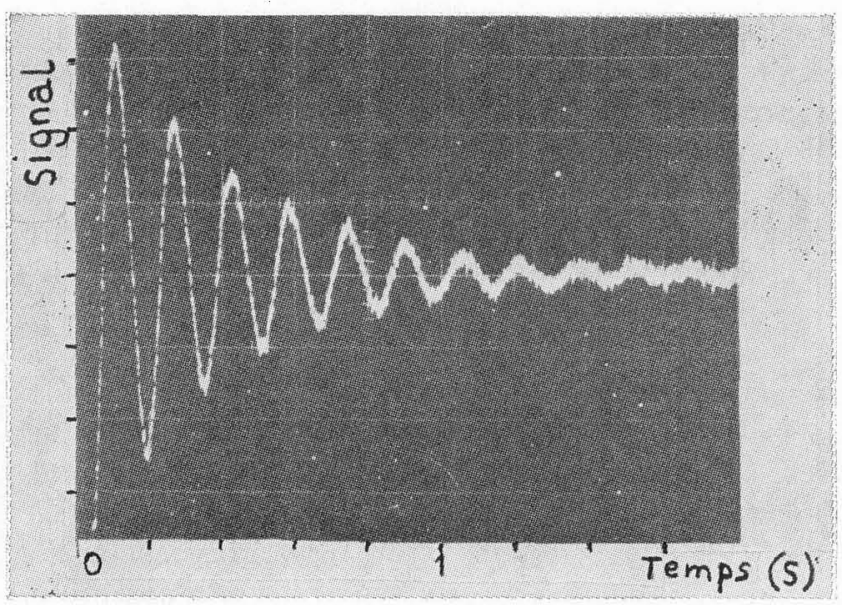

FIG. 11. - Précession de Larmor d'atomes ${ }^{199} \mathrm{Hg}$ autour du champ fictif $\mathbf{H}_{\mathfrak{q}}$. Résultat expérimental.

Au lieu d'effectuer des expériences en régime transitoire, on peut également laisser les atomes irradiés en permanence par $\mathrm{FL}_{2}$ et leur appliquer en plus un champ statique réel $\mathbf{H}_{0}$, de façon à étudier les modifications qui apparaissent sur leur diagramme Zeeman. Tout se passe comme si les atomes étaient soumis à un champ total $\mathbf{H}_{t}$, somme vectorielle du champ appliqué $\mathbf{H}_{0}$ et du champ fictif $\mathbf{H}_{\mathbf{f}}$ associé à $\mathrm{FL}_{2}$. $\mathrm{Si} \mathbf{H}_{0}$ est appliqué parallèlement à ce faisceau lumineux (Fig. 12a), $\mathbf{H}_{\mathrm{o}}$ et $\mathbf{H}_{\mathrm{f}}$ sont parallèles et le diagramme Zeeman est simplement translaté en champ magnétique : le croisement des 2 sous-niveaux Zeeman apparaît lorsque le champ réel compense le champ fictif, c'est-à-dire lorsque $\mathbf{H}_{0}=-\mathbf{H}_{\mathrm{f}}$.

$\mathrm{Si} \mathbf{H}_{0}$ et $\mathbf{H}_{\mathbf{f}}$ sont perpendiculaires, la direction de $\mathbf{H}_{t}$ varie lorsqu'on balaie $H_{0}$; de plus, $\left|\mathbf{H}_{t}\right|$ ne s'annule jamais et passe par une valeur minimale égale à $\left|\mathbf{H}_{\mathrm{f}}\right|$. Le diagramme Zeeman est formé dans ce cas de 2 branches d'hyperbole admettant pour asymptotes des droites correspondant au diagramme Zeeman de l'atome isolé (Fig. 12b). Dans les 2 cas, la dégénérescence Zeeman est levée en champ statique nul.

Les diagrammes des figures $12 a$ et $12 b$ ont pu être construits expérimentalement [9] en mesurant, pour chaque valeur de $H_{0}$, la distance énergétique séparant les 2 sous-niveaux Zeeman de l'atome irradié par $\mathrm{FL}_{2}$. Les résultats expérimentaux confirment pleinement les prévisions théoriques (Fig. 13).

Lorsque le moment cinétique de l'état fondamental est supérieur ou égal à $\frac{1}{2}$, il faut pour décrire l'effet
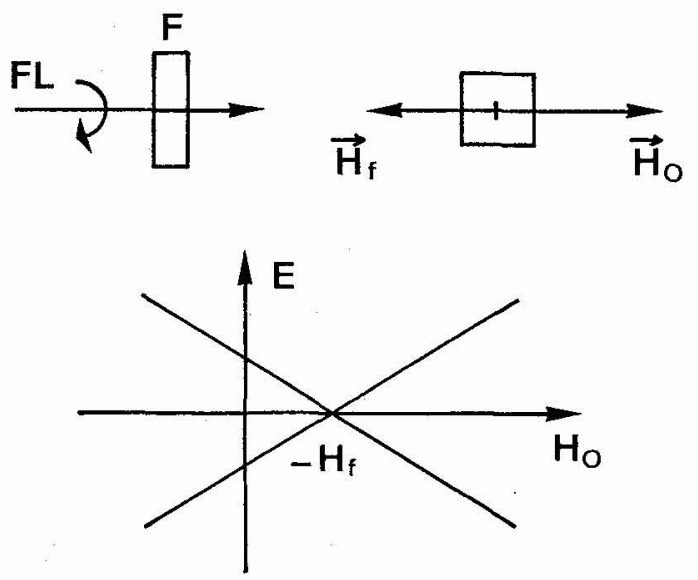

(a)
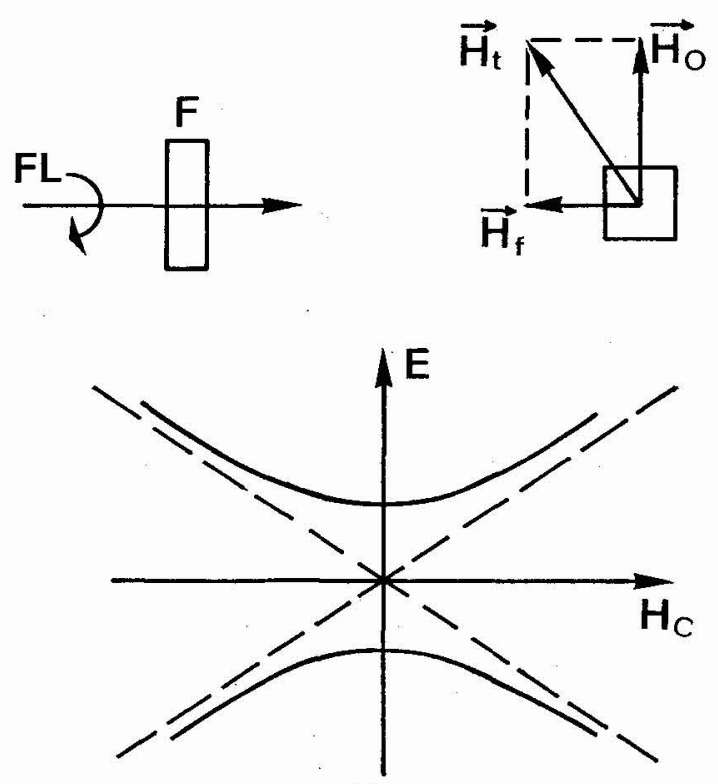

(b)

FIG. 12. - Etude du diagramme Zeeman d'atomes ${ }^{199} \mathrm{Hg}$ soumis à une irradiation lumineuse non résonnante. Schéma de principe.

12a. - Champ statique réel $\mathbf{H}_{0}$ parallèle au champ fictif $\mathbf{H}_{\mathbf{f}}$. 12b. - Champ statique réel $\mathbf{H}_{0}$ perpendiculaire au champ fictif $\mathbf{H}_{\mathrm{p}}$.

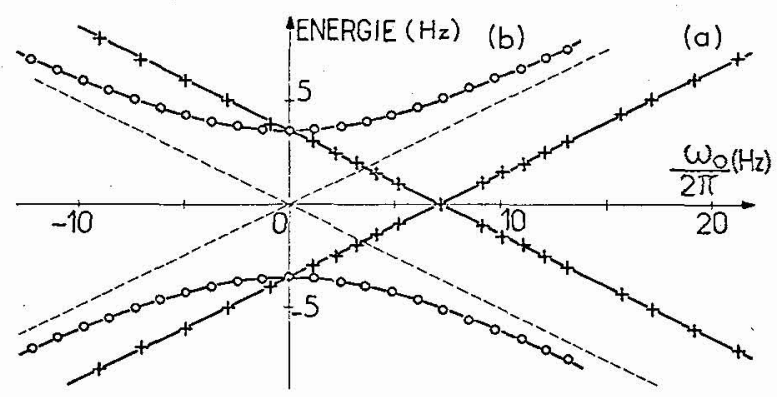

Fig. 13. - Résultats expérimentaux concernant les diagrammes Zeeman d'atomes ${ }^{199} \mathrm{Hg}$ soumis à une irradiation lumineuse non résonnante. Traits pointillés : diagramme Zeeman de l'atome libre; Traits pleins : courbes théoriques ; $O$ : Résultats expérimentaux correspondant à $\mathbf{H}_{0} \perp \mathbf{H}_{\mathrm{f}} ;+$ : Résultats expérimentaux correspondant à $\mathbf{H}_{0} / / \mathbf{H}_{\mathrm{p}}$. 

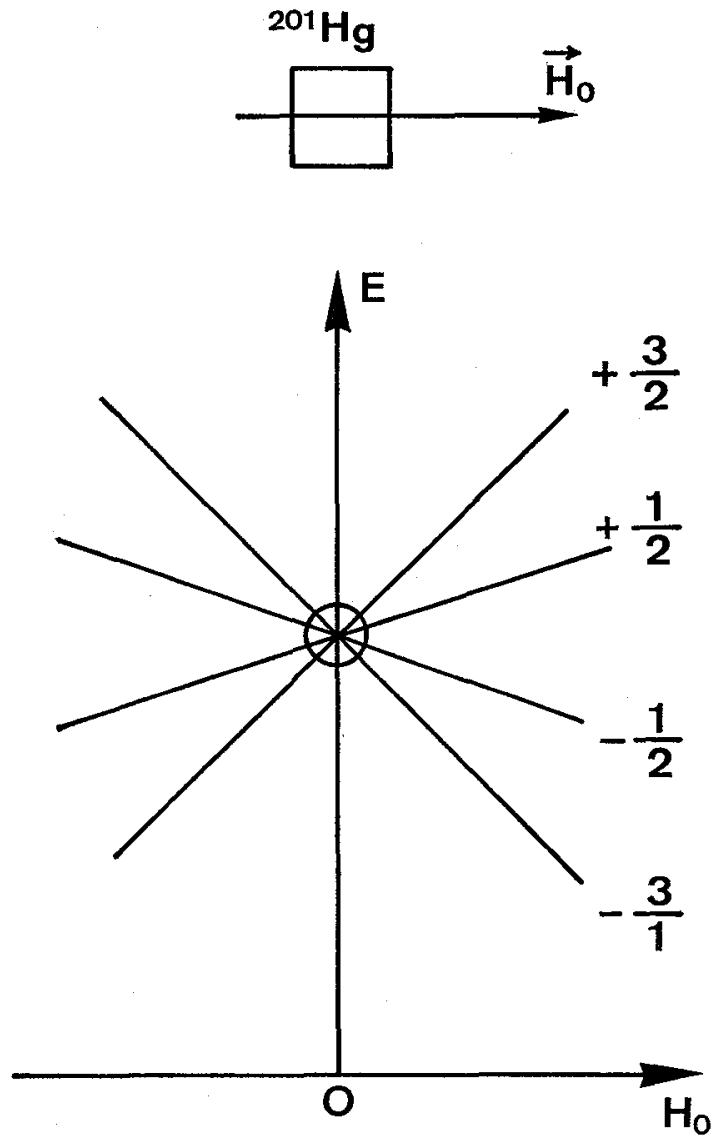

(a)
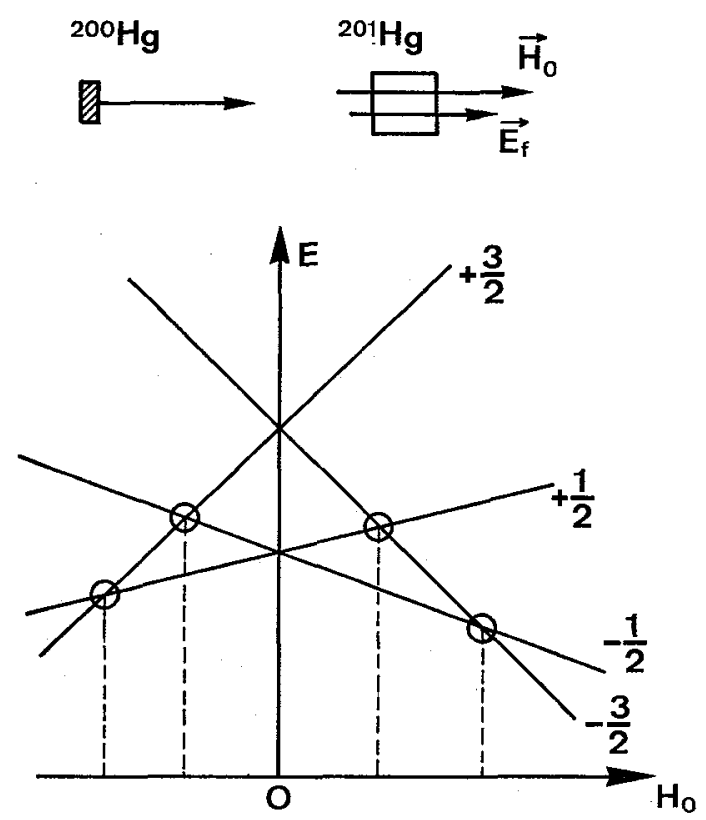

(b)

FIG. 14.

$14 a_{6}$ - Diagramme Zeeman d'atomes ${ }^{201} \mathrm{Hg}$ libres.

14b. - Diagramme Zeeman d'atomes $201 \mathrm{Hg}$ soumis à une irradiation lumineuse non résonnante (champ électrique fictif $\mathbf{E}_{\mathfrak{f}}$ parallèle à $\mathbf{H}_{0}$ ). Schéma de principe. de l'irradiation lumineuse non résonnante introduire, en plus du champ magnétique statique fictif $\mathbf{H}_{f}$, un champ électrique statique fictif $\mathbf{E}_{\mathrm{f}}[10]$.

$\mathrm{La}$ figure 14 représente les diagrammes Zeeman de l'état fondamental d'atomes ${ }^{201} \mathrm{Hg}\left(I=\frac{3}{2}\right)$ isolés (Fig. 14a), et irradiés par un faisceau lumineux non polarisé et non résonnant (issu d'une lampe à ${ }^{200} \mathrm{Hg}$ ) se propageant le long du champ statique $\mathbf{H}_{0}$ (Fig. 14b). On peut montrer que l'effet de ce faisceau lumineux est équivalent à celui d'un champ électrique statique fictif $\mathbf{E}_{\mathrm{f}}$ parallèle à la direction de propagation $\mathrm{du}$ faisceau. L'effet Stark qui lui est associé déplace de la même quantité les niveaux $+\frac{3}{2}$ et $-\frac{3}{2}$ d'une part, $+\frac{1}{2}$ et $-\frac{1}{2}$ d'autre part, ce qui donne naissance au diagramme de la figure $14 b$, où l'on voit apparaître 4 croisements de niveaux en champ non nul (entourés par un rond).

Ces croisements de niveaux peuvent être observés dans des expériences de pompage optique transversal [11] car on peut montrer qu'il apparaît sur les signaux de détection optique des résonances lorsqu'on balaie le champ statique au voisinage des valeurs correspondant à ces croisements. La figure 15 montre les signaux obtenus en l'absence et en présence du faisceau non résonnant. On voit bien apparaitre les 4 croisements du diagramme de la figure $14 b$ (les croisements qui subsistent en champ nul sont inobservables dans les conditions de l'expérience).

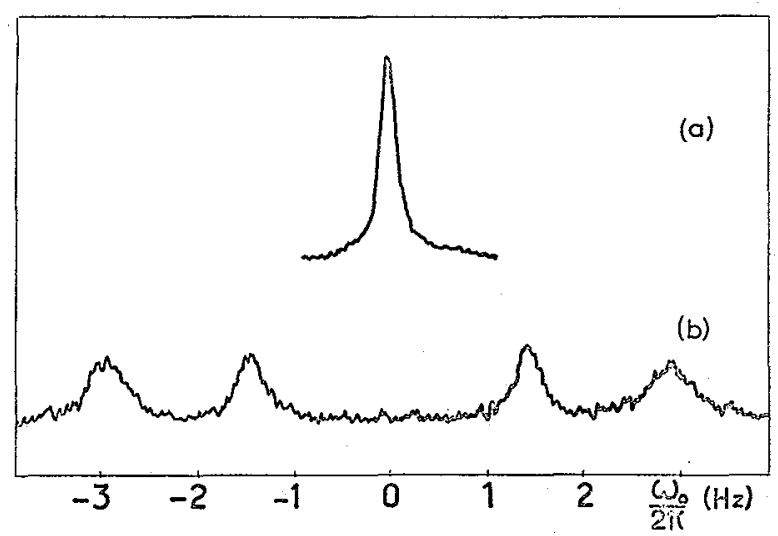

FIG. 15. - Résultats expérimentaux correspondant aux diagrammes de la figure 14.

15a. - Résonance correspondant au croisement de niveaux de la figure $14 a$.

15b. - Résonances correspondant aux 4 croisements de niveaux (entourés d'un rond) de la figure $14 b$.

Un autre exemple intéressant est celui de ${ }^{87} \mathrm{Rb}$ qui possède 2 niveaux hyperfins $F=1$ et $F=2$ dans l'état fondamental. La figure $16 a$ représente le diagramme Żeeman de l'atome ${ }^{87} \mathrm{Rb}$ isolé. Irradions la cellule de ${ }^{87} \mathrm{Rb}$ par un faisceau non résonnant, polarisé circulairement, issu d'une lampe à ${ }^{85} \mathrm{Rb}$, et se propageant le long de $\mathbf{H}_{0}$ (Fig. $16 b$ ) ; le filtre à ${ }^{87} \mathrm{Rb}$ sert à éliminer les fréquences résonnantes. L'effet de cette irradiation lumineuse est plus complexe à décrire 


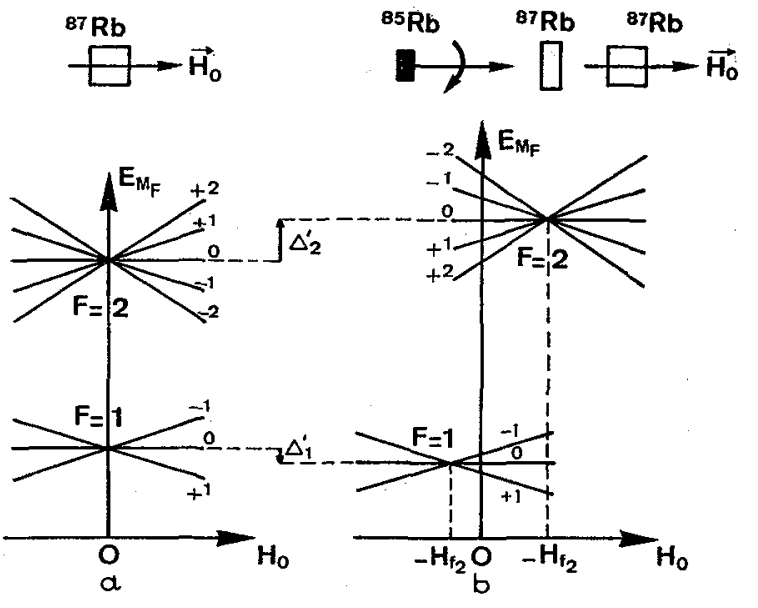

F1G. 16.

16a. - Diagramme Zeeman d'atomes ${ }^{87} \mathrm{Rb}$ libres.

16b. - Diagramme Zeeman d'atomes ${ }^{87} \mathbf{R b}$ soumis à une irradiation lumineuse non résonnante. Schéma de principe.

(Les champs fictifs $\mathbf{H}_{P_{1}}$ et $\mathbf{H}_{q_{2}}$ décrivant l'effet de cette irradiation

à l'intérieur des niveaux $\mathrm{F}=1$ et $\mathrm{F}=2$ sont opposés.)

que dans les 2 cas précédents : les niveaux $F=2$ et $\mathrm{F}=1$ sont déplacés en bloc dans des sens opposés et de quantités respectivement égales à $\Delta_{2}^{\prime}$ et $\Delta_{1}^{\prime}$; de plus, tout se passe comme si l'on soumettait les niveaux hyperfins à des champs fictifs $\mathbf{H}_{\mathrm{f}_{1}}$ et $\mathbf{H}_{\mathrm{f}_{2}}$, de grandeurs différentes et de sens opposés. Il en résulte

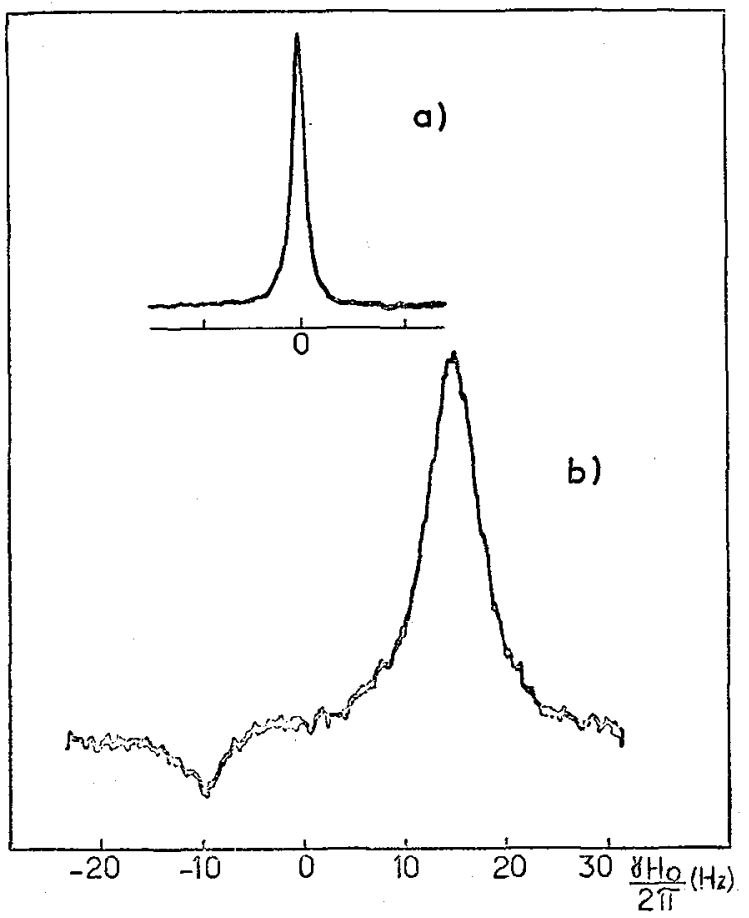

Fig. 17. - Résultats expérimentaux correspondant aux diagrammes de la figure 16.

17a. - Résonance associée aux croisements de niveaux de la figure $16 a$ (qui coincident en champ nul).

17b. - Résonances associées aux 2 croisements de niveaux de la figure $16 h$. le diagramme Zeeman de la figure $16 b$. La fréquence de la raie $0-0\left(\mathrm{~F}=2, M_{\mathrm{f}}=0 \leftrightarrow \cdots \mathrm{F}=1, M_{\mathrm{f}}=0\right)$, qui joue un rôle fondamental dans les horloges atomiques puisque sa fréquence ne dépend pas de $H_{0}$ au $1^{\text {er }}$ ordre est déplacée d'une quantité $\Delta_{2}^{\prime}-\Delta_{1}^{\prime}$. Cet effet a été observé expérimentalement [12] et étudié en grand détail [13] puisqu'il constitue une cause possible d'erreur sur la fréquence délivrée par une horloge atomique. L'effet de $\mathbf{H}_{\mathrm{f}_{2}}$ et $\mathbf{H}_{\mathrm{f}_{1}}$ a été observé plus récemment [14]. Il donne naissance à 2 résonances de croisement de niveaux apparaissant pour $H_{0}=-H_{\mathrm{f}_{1}}$ et $H_{0}=-H_{\mathrm{f}_{2}}$ (Fig. 17).

L'exemple précédent montre clairement le caractère fictif du champ $\mathbf{H}_{\mathrm{f}}$ puisque, pour une même irradiation lumineuse, ce champ varie d'un niveau hyperfin à l'autre. C'est là d'ailleurs l'un des intérêts de ces champs fictifs puisque, dans un mélange d'éléments, ils peuvent agir sélectivement sur certains éléments et sur certains niveaux seulement. Mentionnons enfin qu'on peut, en modulant l'intensité ou la polarisation du faisceau lumineux non résonnant, soumettre un atome à un champ fictif électrique ou magnétique oscillant; ceci permet par suite d'induire des transitions résonnantes entre certains des sous-niveaux Zeeman, lorsque la fréquence de modulation précédente coïncide avec l'une des fréquences hertziennes de l'atome [15], [16].

B. Interaction d'un atome avec des photons de radiofréquence non résonnants. - L'étude précédente montre que le diagramme Zeeman d'un atome peut être passablement modifié lorsqu'on « habille» cet atome avec des photons optiques non résonnants. Nous allons voir maintenant que des photons de radiofréquence $(R F)$ non résonnants peuvent également produire des changements tout aussi importants.

1. ETATS dU Système Global ATOME + Champ $R F$. - Considérons donc un spin $\frac{1}{2}$, I, plongé dans un champ statique $\mathbf{H}_{0}$ parallèle à $\mathrm{O} z$ et un champ $R F$ de pulsation $\omega$ dont nous ne précisons pas pour l'instant la polarisation.

En l'absence de tout couplage entre les 2 systèmes, les états d'énergie du spin sont les états propres $\mid+>$ et $\mid->$ de $I_{z}$, d'énergie $+\omega_{0} / 2$ et $-\omega_{0} / 2$ avec

$$
\omega_{0}=-\gamma H_{0} .
$$

Les états du champ $R F$ sont les états $|n\rangle$ comprenant $n$ photons, et d'énergie $n \omega$ (dans le domaine des $R F$, on peut négliger le demi-quantum $\frac{1}{2} \omega$ ). Les états $1 \pm, n>$ du système global sont donc repérés par 2 nombres quantiques et correspondent à une situation où le spin est dans l'état $\mid \pm>$ en présence de $n$ photons, l'énergie non perturbée du système étant égale à $\pm \omega_{0} / 2+n \omega$.

Il peut paraître surprenant de quantifier un champ $R F$ qui est essentiellement classique. En fait, en procédant ainsi, on traite le champ non comme une per- 
turbation oscillante imposée de l'extérieur, mais comme une partie d'un système global, l'atome + le champ $R F$, qui est isolé et qui est par suite décrit par un hamiltonien indépendant du temps [17]. Les calculs s'en trouvent souvent simplifiés par rapport à ceux de la théorie classique qui fait intervenir un hamiltonien dépendant du temps. En particulier, la quantification $\mathrm{du}$ champ réalise d'emblée, et pour une polarisation quelconque de la radiofréquence, l'équivalent du passage dans le référentiel tournant de la théorie classique qui consiste précisément à éliminer la dépendance temporelle de l'hamiltonien et qui n'est valable que pour un champ $R F$ tournant. De plus, l'adoption dès le départ d'un point de vue quantique permet de préciser clairement le rôle des photons $R F$ dans les divers effets observés.

Pour étudier le couplage entre l'atome et le champ, nous supposerons que ce dernier a une polarisation linéaire et parallèle à $O x$, c'est-à-dire perpendiculaire à $\mathbf{H}_{0}$ (Fig. 18). L'hamiltonien d'interaction $V$ est proportionnel au produit scalaire du moment ciné-

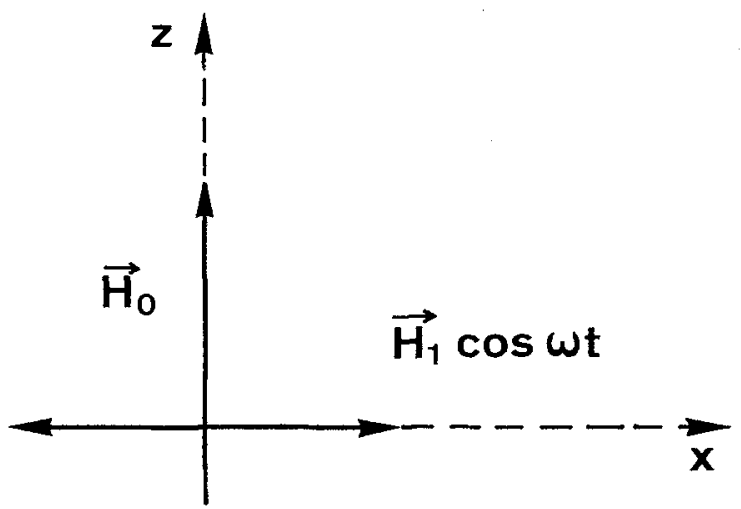

FIg. 18. - Disposition relative du champ statique $\mathbf{H}_{0}$ et du champ $R F \mathbf{H}_{1} \cos \omega t$.

tique I par l'opérateur champ magnétique de radiofréquence. La polarisation de ce dernier étant parallèle à $\mathrm{O} x$, il s'ensuit que $V$ est proportionnel à $I_{x}$ et ne peut par suite coupler l'état $\mid+>$ qu'à l'état $\mid->$ et réciproquement :

$$
1+>\leftrightarrow \cdots+->\text {. }
$$

D'autre part, l'opérateur champ magnétique est une combinaison linéaire des opérateurs de création et d'annihilation d'un photon, $\mathrm{a}^{+}$et $\mathrm{a}$, et ne peut par suite coupler l'état $|\mathrm{n}\rangle$ qu'aux états $|\mathrm{n}+1\rangle$ et $|\mathrm{n}-1\rangle$ :

$$
|n-1>\leftrightarrow-\cdots| n>\cdots|n+1\rangle \text {. }
$$

On en déduit très simplement les règles de sélection auxquelles satisfait l'hamiltonien d'interaction $V$. $V$ ne couple l'état $\mid+, \mathbf{n}>$ qu'aux 2 états $\mid-, \mathrm{n}+1>$ et $\mid-, \mathrm{n}-1>$, l'état $\mid-, \mathrm{n}>$ qu'aux 2 états

$\mid+, \mathrm{n}+1>$ et $\mid+; \mathrm{n}-1>$ :

$|-, \mathrm{n}-1>\leftarrow-\cdot|+, \mathrm{n}>\cdots \mid-, \mathrm{n}+1>$

$|+, \mathrm{n}-1>\leftrightarrow--|-, \mathrm{n}>\cdots+\cdots \mid+, \mathrm{n}+1>$

l'interprétation physique de ces règles de sélection est très claire : les seuls processus élémentaires possibles correspondent au passage de l'atome d'un sous-niveau Zeeman à l'autre par émission induite ou absorption d'un photon $R F$.

On peut aisément s'assurer que le moment cinétique global est conservé au cours de tels processus. Si l'énergie globale est également conservée, c'est-à-dire si le champ $R F$ est résonnant $\left(\omega=\omega_{0}\right)$, on a affaire à des transitions réelles de résonance magnétique qui jouent un rôle très important en spectroscopie hertzienne. Si l'on est hors de résonance $\left(\omega \neq \omega_{0}\right)$, les processus d'absorption et d'émission induite précédents deviennent virtuels mais peuvent cependant conduire à des effets observables.

2. Moment MagnétiQue de L'ATOME habillé PaR DES PHOTONS RF [18]. - La figure 19 représente

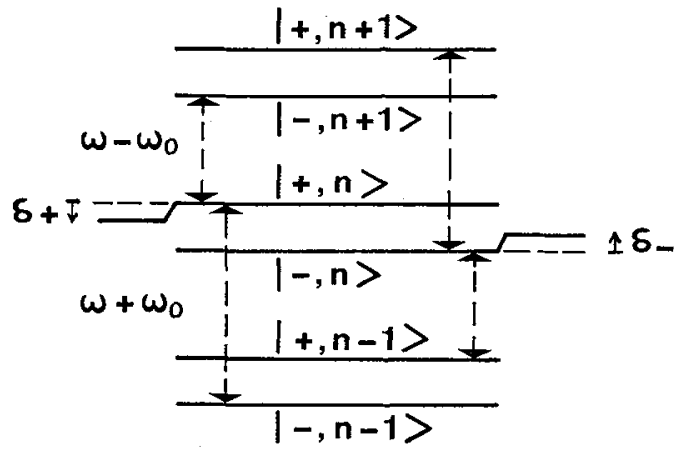

Fig. 19. - Déplacement des niveaux $\mid+, \mathrm{n}>$ et $\mid-, \mathrm{n}>$ sous l'effet du couplage $V$ entre l'atome et le champ $R F$. Les flèches relient les états qui sont couplés par $V$.

les niveaux d'énergie non perturbés du système global atome + champ $R F$ en champ statique très faible

$$
\omega_{0} \ll \omega \text {. }
$$

Ils se groupent en multiplicités correspondant à un même nombre de photons $n$, la distance $\omega$ entre multiplicités étant grande devant l'écart $\omega_{0}$ qui sépare 2 états d'une même multiplicité. Les 2 flèches partant de l'état $\mid+, n>$ indiquent les états $\mid-, n+1>$ et $|-, \mathrm{n}-1\rangle$ auxquels ce dernier est couplé par $V$ et qui sont à des distances respectivement égales à $\omega-\omega_{0}$ et $\omega+\omega_{0}$. Les éléments de matrice correspondants de $V$ sont pratiquement égaux (dans la mesure où l'on peut confondre $\sqrt{\mathrm{n}+1}$ et $\sqrt{\mathrm{n}}$ lorsque 
$\mathrm{n}$ est très grand), et leur valeur commune $K$ peut être reliée très aisément au paramètre

$$
\omega_{1}=-\gamma H_{1}
$$

qui dans la théorie classique repère la force du couplage. $\left(\omega_{1}\right.$ est la pulsation de Larmor autour du champ $\boldsymbol{R} F$ classique $\mathbf{H}_{1} \cos \omega t$. Voir figure 18.) On trouve :

$$
K=\frac{\omega_{1}}{4} .
$$

Les 2 éléments de matrice de $V$ partant de l'état $\mid-, \mathrm{n}>$ sont eux aussi égaux à $K$.

Si nous supposons le couplage faible, c'est-à-dire si

$$
\omega_{1} \ll \omega
$$

on peut utiliser la théorie des perturbations pour évaluer le déplacement de l'état $|+, \mathrm{n}\rangle$. On sait qu'un couplage entre 2 états d'énergie différents les fait se repousser, d'autant plus efficacement que les 2 états sont rapprochés. L'état $|+, \mathrm{n}\rangle$ est donc repoussé vers le bas par l'état $|-, n+1\rangle$, vers le haut par l'état $|-, n-1\rangle$. Comme l'état $\mid-, n+1>$ est le plus proche et que les divers éléments de matrice de $V$ sont égaux, on en conclut que le déplacement global $\delta_{+}$de l'état $\mid+, \mathrm{n}>$ se fait vers le bas et est donné par l'expression

$$
\begin{aligned}
\delta_{+}=-K^{2}\left[\frac{1}{\omega-\omega_{0}}-\frac{1}{\omega+\omega_{0}}\right] & = \\
=-K^{2} \frac{2 \omega_{0}}{\omega^{2}-\omega_{0}^{2}} & \simeq-\frac{\omega_{1}^{2}}{8 \omega^{2}} \omega_{0} .
\end{aligned}
$$

On a utilisé (18). $\delta_{+}$est donc proportionnel à $\omega_{0}$. Un raisonnement identique permettrait de montrer que l'état $|-, \mathrm{n}\rangle$ est déplacé vers le haut d'une quantité $\delta_{-}$, égale à $-\delta_{+}$, ce qui fait finalement passer l'écart entre les 2 niveaux $|+, \mathrm{n}\rangle$ et $1-, \mathrm{n}>$ de la valeur $\omega_{0}$ à la valeur

$$
\omega_{0}\left(1-\frac{\omega_{1}^{2}}{4 \omega^{2}}\right)
$$

Le facteur de Landé de l'atome est donc multiplié par le facteur

$$
1-\frac{\omega_{1}^{2}}{4 \omega^{2}}
$$

sous l'effet des absorptions et réémissions induites virtuelles (ou encore des émissions induites et réabsorptions virtuelles) de photons $R F$.

Si l'on augmente le nombre moyen de photons, c'est-à-dire encore l'énergie totale contenue dans le champ $R F$, le couplage $V$ ne peut plus être traité comme une perturbation : $\omega_{1}$ devient comparable ou supérieur à $\omega$. Il est encore possible cependant de calculer le facteur de Landé $g_{\mathrm{h}}$ de l'atome habillé par les photons $R F$. L'hamiltonien du système global s'écrit en effet

$$
\mathscr{H}=\mathscr{H}_{z}+\mathscr{H}_{R F}+V .
$$

Si le terme de couplage $V$ n'est plus négligeable devant les 2 autres, on peut au contraire traiter le terme Zeeman $\mathfrak{H}_{z}$, qui décrit le couplage du spin avec le champ statique, comme une perturbation vis-à-vis de $\mathscr{H}_{R F}+V\left(\mathfrak{H}_{R F}\right.$ représente l'énergie propre du champ $\left.R F\right)$. Il se trouve que l'on sait trouver exactement les états propres et valeurs propres de l'hamiltonien $\mathfrak{H}_{R F}+V$ [19]. Ses niveaux se groupent en multiplicités doublement dégénérées et distantes de $\omega . \mathfrak{H}_{z}$ lève cette dégénérescence, et le calcul des énergies à l'ordre 1 en $\mathfrak{H}_{z}$, c'est-à-dire en $\omega_{0}$, donne la pente en champ statique faible des niveaux d'énergie du système global atome + champ $R F$, c'est-à-dire encore le facteur de Landé $g_{h}$ de l'atome habillé. Un calcul simple permet ainsi d'obtenir pour $g_{\mathrm{h}}$ l'expression :

$$
g_{\mathbf{h}}=g_{0} J_{0}\left(\frac{\omega_{1}}{\omega}\right)
$$

où $g_{0}$ est le facteur de Landé de l'atome isolé, et $J_{0}$ la fonction de Bessel d'ordre 0 [18]. On vérifie immédiatement qu'un développement limité de (23) redonne le résultat trouvé en (21) lorsque le couplage $\omega_{1} / \omega$ est petit.

On voit donc que le facteur de Landé de l'atome habillé par des photons $R F$ polarisés linéairement peut être considérablement modifié par rapport à celui de l'atome isolé. Il peut même s'annuler pour les valeurs de $\omega_{1} / \omega$ correspondant à un zéro de $J_{0}$. Ainsi l'effet "stimulé » que nous mettons ici en évidence peut être plus grand que l'effet "spontané » de modification du facteur $g$ del'électron (anomalie $\mathrm{g}-2$ ), que nous avons rappelé dans l'introduction et qui est de l'ordre du millième. Ceci n'est pas surprenant puisque, dans le domaine des $R F$, l'absorption et l'émission induite peuvent l'emporter aisément sur l'émission spontanée. Une autre différence entre les 2 types d'effets provient de l'anisotropie introduite par la direction privilégiée de la polarisation du champ $R F$. Lorsqu'on étudie l'évolution de $\langle\mathbf{I}\rangle$ en présence du couplage, et en tenant compte de la modification des états propres du système global, on trouve que l'extrémité du moment cinétique atomique décrit autour de $\mathbf{H}_{0}$, non pas un cercle comme c'est le cas pour un atome isolé, mais une ellipse d'excentricité $J_{0}\left(\omega_{1} / \omega\right)$ et dont le grand axe est parallèle à la polarisation du champ $R F$ [20].

Tous ces effets ont été observés expérimentalement. Le principe des expériences est schématisé sur la figure 20. Dans une première étape (Fig. 20a), on oriente les spins atomiques en champ statique nul dans la direction $O x$ au moyen d'un faisceau de pompage optique se propageant le long de cette direction. La mesure de la lumière transmise au moyen 


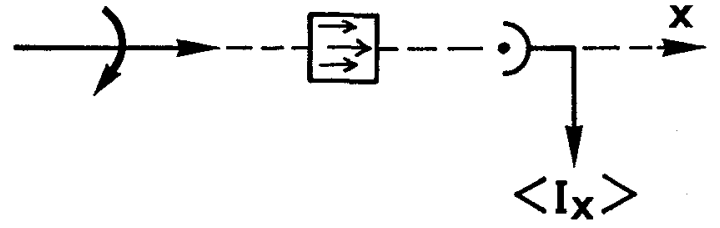

(a)

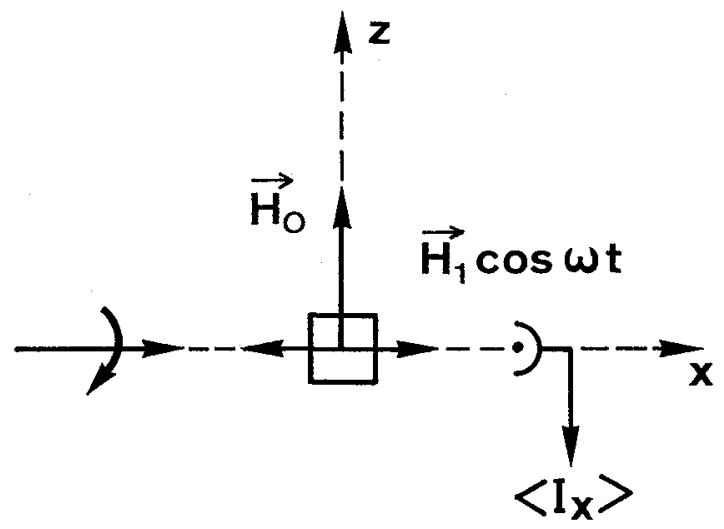

(b)

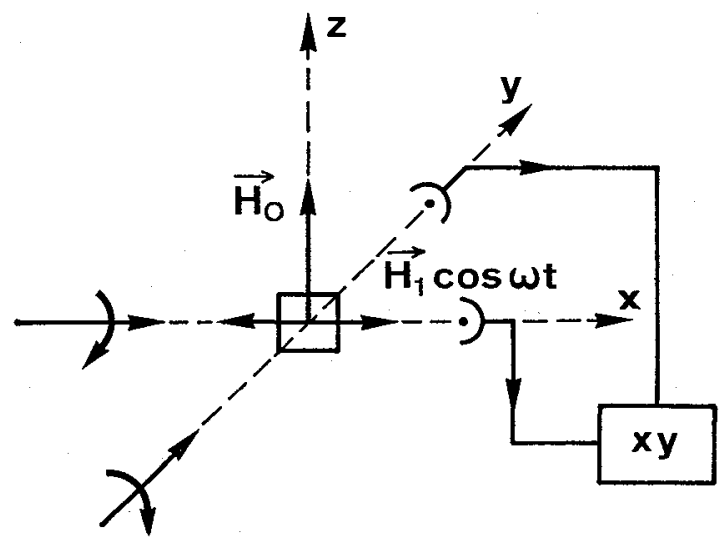

(c)

Fig. 20. - Dispositif expérimental pour l'étude de la précession de Larmor de l'atome habillé par des photons $R F$. Schéma de principe.

d'un photomultiplicateur fournit un signal proportionnel à $\left\langle I_{x}\right\rangle$. Introduisons alors brusquement le champ statique $\mathbf{H}_{0}$ parallèlement à $\mathrm{O} z$ et le champ $R F$ parallèlement à $\mathrm{O} x$ (Fig. 20b). Les spins initialement orientés le long de $\mathrm{O} x$ se mettent à précesser autour de $\mathbf{H}_{0}$ avec une vitesse angulaire $\bar{\omega}_{0}$ qui diffère de celle de l'atome isolé par le facteur $J_{0}\left(\omega_{1} / \omega\right)$. Il en résulte une modulation de la lumière transmise à la fréquence $\bar{\omega}_{0} / 2 \pi$, ce qui permet ainsi de déterminer $\bar{\omega}_{0}$. La figure 21 montre les modulations obtenues lorsqu'on recommence l'expérience avec la même valeur de $H_{0}$ mais des valeurs différentes de $H_{1}$, donc de $\omega_{1} / \omega$ [20]. On voit clairement que la précession est plus lente lorsque $\omega_{1} / \omega$ augmente, disparaît complètement pour $\omega_{1} / \omega=2,4$ (valeur correspondant au $1^{\text {er }}$ zéro de la fonction de Bessel $J_{0}$ : fig. $21 f$ ), puis croît à nouveau.

On peut également mettre en évidence l'ellipticité de la précession de Larmor. Le principe général de l'expérience est le suivant (Fig. 20c) : En même temps que l'on introduit le champ statique et le champ $R F$, on démasque un second faisceau lumineux de détection, se propageant le long de $\mathrm{O} y$ et d'intensité suffisamment faible pour ne pas trop amortir la précession de spins. La mesure de la lumière transmise fournit un signal proportionnel à $\left\langle I_{y}\right\rangle$ que l'on peut envoyer sur un enregistreur $X Y$ en même temps que le signal obtenu sur le $1^{\text {er }}$ faisceau et qui est proportionnel à $\left\langle I_{x}\right\rangle$. On reconstruit ainsi directement la précession de Larmor.

La figure 22 représente les résultats obtenus [20]. L'expérience est d'abord réalisée en l'absence de champ $R F$ et le gain sur les 2 voies $\mathrm{X}$ et $\mathrm{Y}$ ajusté de façon que la précession obtenue soit circulaire. Elle est en fait amortie par la relaxation thermique. Lorsqu'on introduit le champ $R F$, on constate que la précession est ralentie : pendant le même temps de relaxation, on obtient un moins grand nombre de tours. De plus, elle devient anisotrope. Lorsque $\omega_{1} / \omega=2,4$, il n'y a plus du tout de précession.

La possibilité de faire varier le moment magnétique d'un atome en l'habillant avec des photons $R F$ peut conduire à des applications intéressantes.

La figure $23 a$ représente par exemple les diagrammes Zeeman de l'état fondamental d'un atome d'hydrogène isolé. 2 transitions hyperfines $\Delta m_{\mathrm{f}}= \pm 1$ relient le niveau hyperfin $F=0$ au niveau hyperfin $F=1$ et donnent naissance à 2 raies séparées dans un champ $H_{0}$ par l'intervalle $2 \omega_{0}$. Si l'on habille l'atome avec des photons $R F$, la pente des niveaux $\mathrm{F}=1, m_{\mathrm{f}}= \pm 1$ diminue par le facteur $J_{0}\left(\omega_{1} / \omega\right)$ (Fig. 23b). Dans le même chàmp $H_{0}$, les 2 raies précédentes se rapprochent et ne sont plus séparées que par un intervalle

$$
2 \bar{\omega}_{0}=2 \omega_{0} J_{0}\left(\frac{\omega_{1}}{\omega}\right) \text {. }
$$

L'effet précédent a été observé sur un maser à hydrogène fonctionnant sur la raie $0,0 \rightarrow 1,1$ [21]. Il apparaît également sur le spectre hyperfin de ${ }^{87} \mathbf{R b}$. On observe alors non pas 2 mais 4 raies $\Delta m_{\mathrm{f}}= \pm 1$ qui se rapprochent lorsque $\omega_{1} / \omega$ augmente, pour venir se confondre lorsque $\omega_{1} / \omega=2,4$ puis se séparer à nouveau (Fig. 24).

Dans les 2 cas précédents $\left({ }^{87} \mathrm{Rb}\right.$ et $\left.\mathrm{H}\right)$, l'étude des variations avec $\omega_{1} / \omega$ du rapport entre l'écart (S) des raies correspondant à l'atome habillé et celui $\left(\mathrm{S}_{0}\right)$ correspondant à l'atome isolé confirme pleinement les prévisions théoriques représentées par la courbe en trait plein de la figure 25 [21].

3. Diagrammes Zeeman de l'atome habillé. Nous nous sommes limités jusqu'ici à l'étude des propriétés magnétiques de l'atome «habillé » en champ faible. On peut également, en suivant la variation 


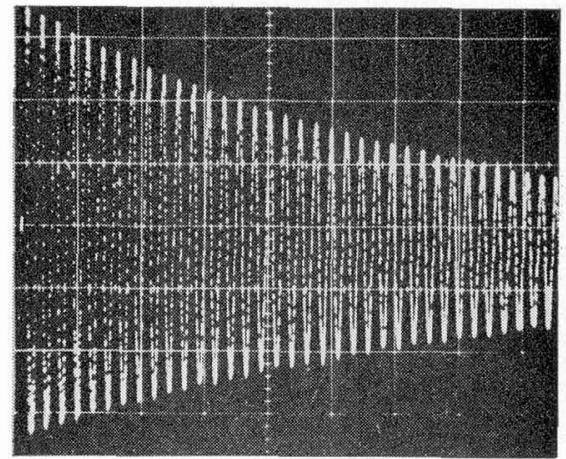

(a)

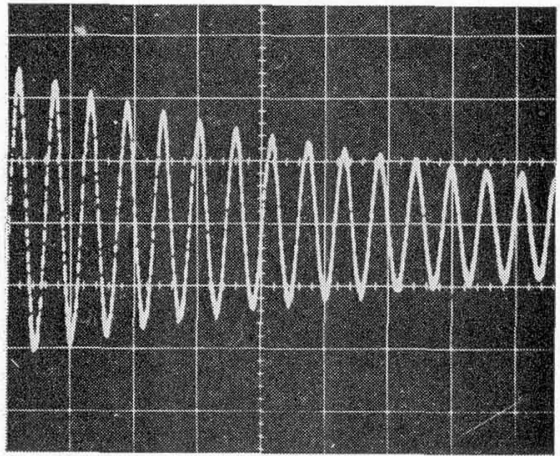

(b)

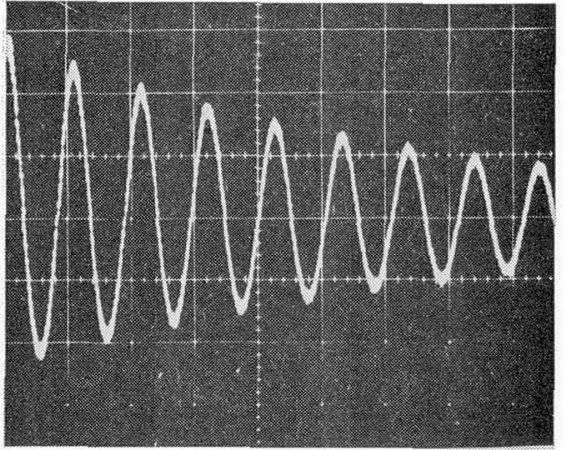

(c)

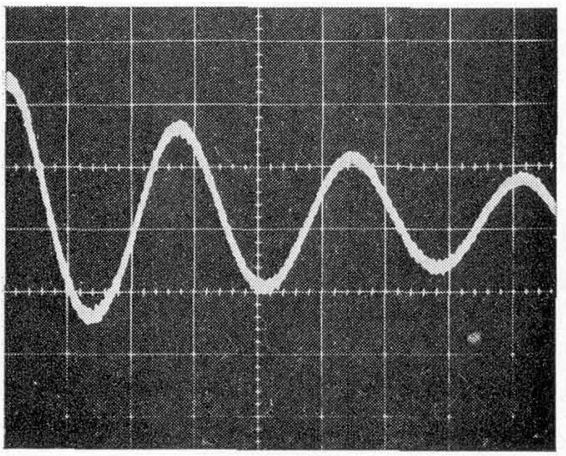

(d)

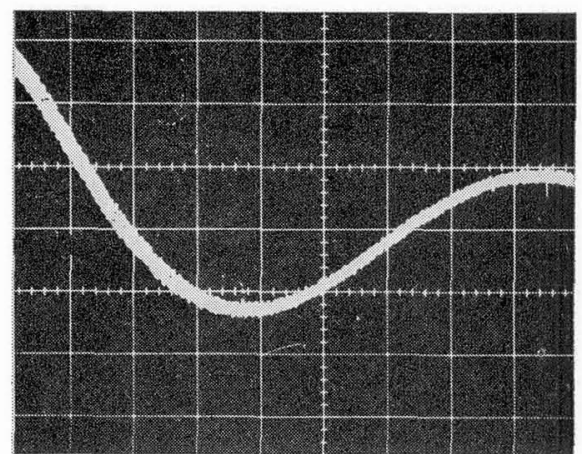

$(e)$
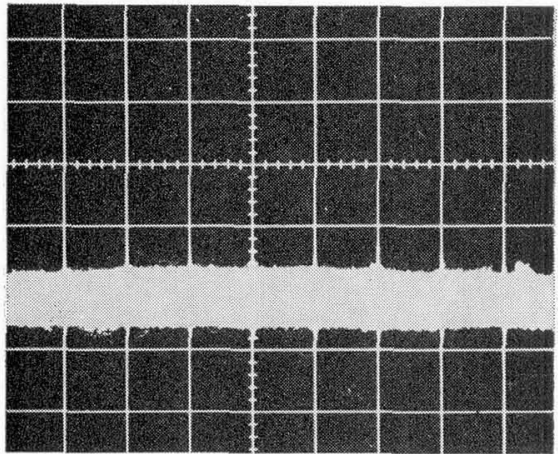

$(f)$

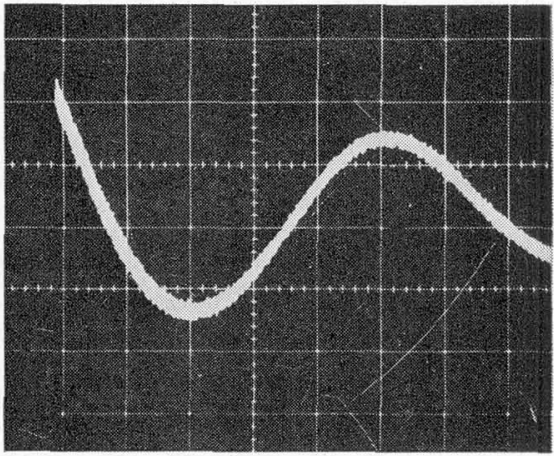

$(g)$

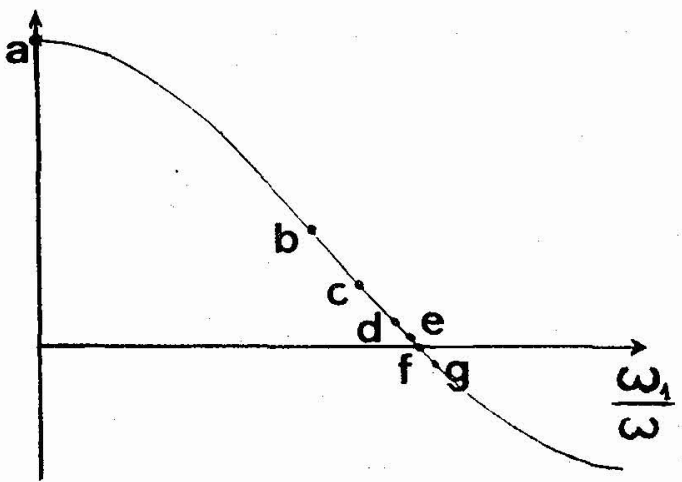

(h)

FIG. 21. - Résultats expérimentaux concernant la fréquence de Larmor d'atomes ${ }^{199} \mathrm{Hg}$ habillés par des photons $R F$. Chaque transitoire correspond à une valeur donnée de $\omega_{1} / \omega$ repérée sur la figure en bas à droite. 


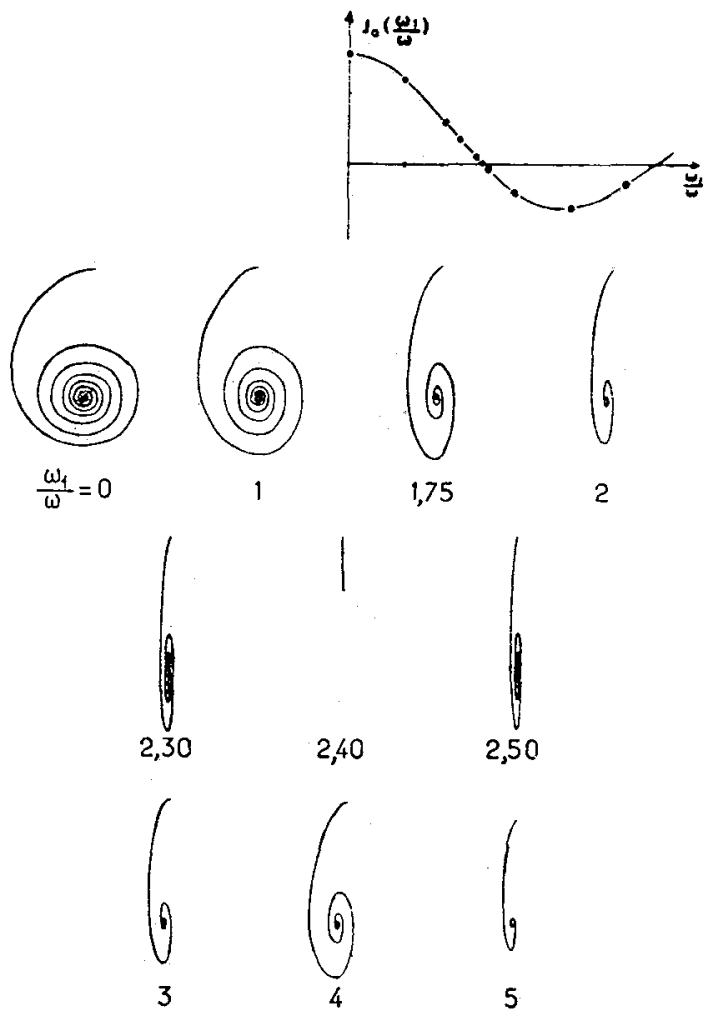

Fig. 22. - Résultats expérimentaux concernant l'ellipticité de la précession de Larmor d'atomes ${ }^{199} \mathrm{Hg}$ habillés par des photons $R F$. Le chiffre figurant au-dessous de chaque transitoire indique la valeur correspondante de $\omega_{1} / \omega$ repérée par ailleurs, sur la figure en haut à droite.

avec $\omega_{0}$ des valeurs propres de l'hamiltonien global $\mathfrak{H}$, déterminer complètement le diagramme Zeeman de l'atome « habillé » [22].

La figure 26 représente par exemple les diagrammes Zeeman d'un spin $\frac{1}{2}$ « habillé » par des photons de radiofréquence de 4 polarisations différentes (Fig. 26a et $26 b$ : champ $R F$ de polarisation linéaire respectivement parallèle et perpendiculaire au champ statique $\mathrm{H}_{0}$; Fig. $26 c$ et $26 d$ : champ $R F$ tournant dans un plan respectivement perpendiculaire et parallèle au champ statique $\mathbf{H}_{0}$ ). Les calculs sont en fait très simples lorsqu'on peut dans (22) traiter $V$ comme une perturbation vis-à-vis du reste, c'est-à-dire lorsque $\omega_{1} / \omega$ est petit $\left({ }^{4}\right)$. Les droites en pointillé de la figure 26 représentent les niveaux non perturbés $\mid \pm, \mathrm{n}>$ introduits plus haut au $\S \mathrm{B} 1$, d'énergie $\mathrm{n} \omega \pm \omega_{0} / 2$. On voit que les diagrammes Zeeman (lignes en traits pleins de la figure 26) présentent en général plusieurs «croisements » et « anticroisements ».

(4) Chacun des diagrammes de la figure 26 présente une périodicité locale : il est invariant lorsqu'on se déplace le long de l'axe des ordonnées d'une quantité égale à $\omega$, ou à un multiple faible de $\omega$ (le nombre de photons $n$ varie alors très peu en valeur relative). Lorsque $n$ varie de façon appréciable en valeur relative l'allure du diagramime change.
On appelle «anticroisement» la figure formée par 2 niveaux qui, au lieu de se croiser en un point, se repoussent parce qu'ils sont couplés soit directement, soit indirectement. Les anticroisements de la figure 26 révèlent donc l'existence d'un couplage entre 2 états différents du système global, de même énergie, et comprenant en général des nombres de photons différents. On peut par conséquent les associer aux résonances à un ou plusieurs quanta [23] (absorption ou émission induite réelle de un ou plusieurs photons $R F$ par l'atome).

Par contre les croisements de la figure 26 correspondent à des niveaux qui ne peuvent être couplés entre eux ni directement ni indirectement (sinon, ils «s'anticroiseraient»). Nous avons déjà montré dans la première partie de cet exposé que l'on pouvait, dans une expérience de pompage optique transversal réalisée sur un atome isolé, obtenir des résonances sur les signaux de détection optique lorsque le champ statique est balayé au voisinage d'un point de croisement de 2 sous-niveaux Zeeman. Le même effet existe sur l'atome "habillé» et des résonances d'un type nouveau, appelées « résonances de cohérence » apparaissent au voisinage de tous les points de croisement de la figure 26. On peut montrer qu'elles sont dues à une variation résonnante des éléments non diagonaux de la matrice densité atomique et qu'elles ne sont observables que par suite d'une contamination des 2 niveaux qui se croisent par des niveaux d'énergie différente et comprenant par suite des nombres de photons différents [17]. Ces résonances sont donc interprétables en termes d'absorptions et d'émissions induites virtuelles de photons $R F$ par l'atome. Elles ont fait l'objet de nombreuses études tant théoriques qu'expérimentales [24].

Les diagrammes Zeeman de l'atome habillé permettent donc d'avoir une vision globale et synthétique des divers effets résonnants de la spectroscopie hertzienne et de préciser le rôle joué par les photons $R F$ dans ces divers effets.

4. UNE APPLICATION PRATIQUE DES RÉSONANCES DE COHÉRENCE : DÉTECTION DE CHAMPS MAGNÉTIQUES TRÈs FAIBLES [25]. - Pour conclure cette étude des effets liés au couplage d'un atome avec des photons optiques ou $R F$ non résonnants, nous citerons une application pratique des résonances de croisements de niveaux de l'atome "habillé» que nous venons juste de décrire.

Lorsque le champ $R F$ a une polarisation linéaire parallèle à $\mathbf{H}_{0}$, une telle résonance existe en champ nul (lorsqu'on balaie $\mathbf{H}_{0}$ autour de la valeur zéro) et peut être détectée sur les modulations qui apparaissent sur les signaux optiques aux différentes harmoniques de $\omega$.

Dansle cas de ${ }^{87} \mathrm{Rb}$, on peut obtenir des résonances particulièrement fines : leur largeur est de l'ordre de $10^{-6} \mathrm{G}$. Une telle finesse est due à la grande valeur $\mathrm{du}$ rapport gyromagnétique de ${ }^{87} \mathrm{Rb}$ et à la longueur 

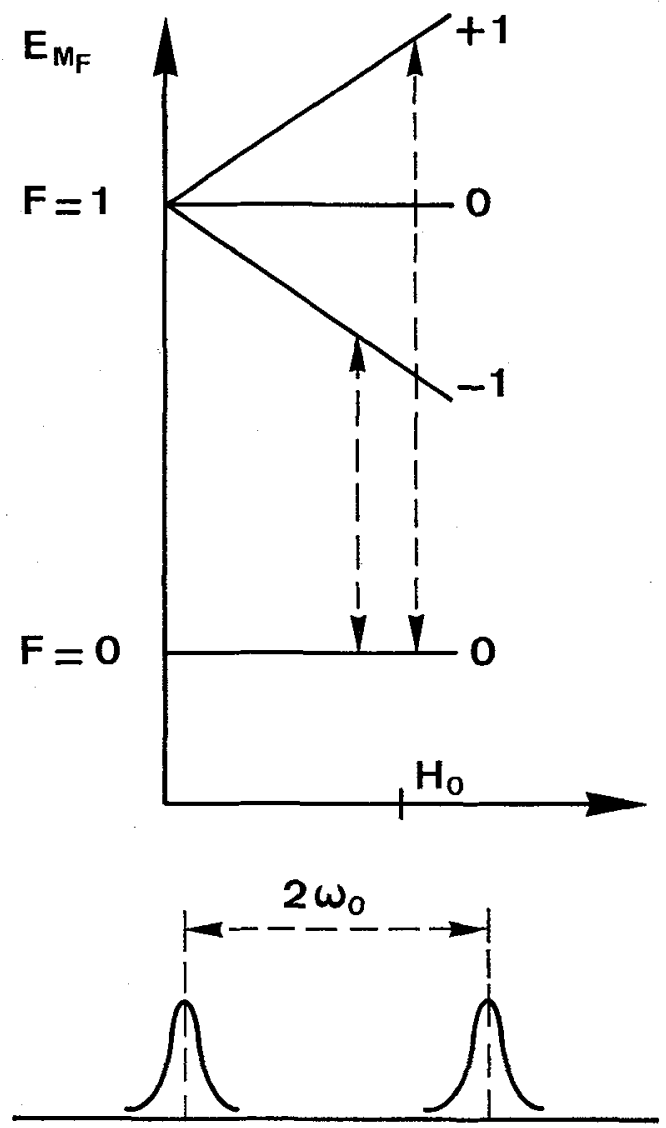

(a)
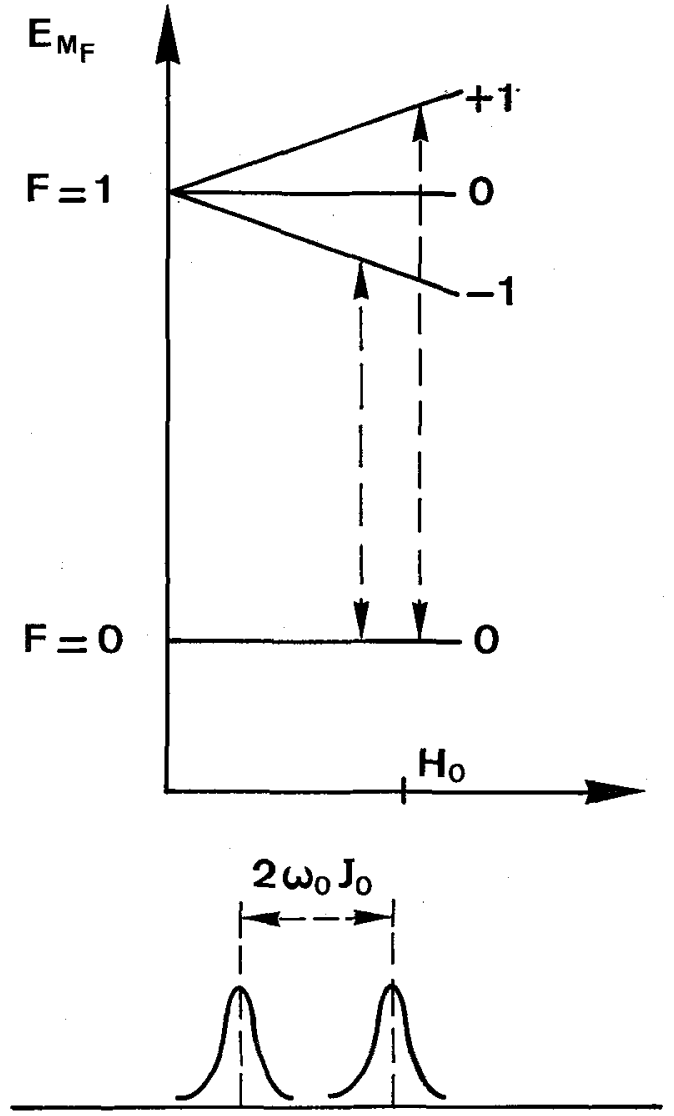

(b)

FIG. 23.

23a. - Spectre hyperfin $\left(\Delta m_{\mathrm{P}}= \pm^{\prime} 1\right)$ d'un atome d'hydrogène libre. Schéma de principe.

23b. - Spectre hyperfin $\left(\Delta m_{\mathrm{f}}= \pm 1\right)$ d'un atome d'hydrogène habillé par des photons $R F$. Schéma de principe.

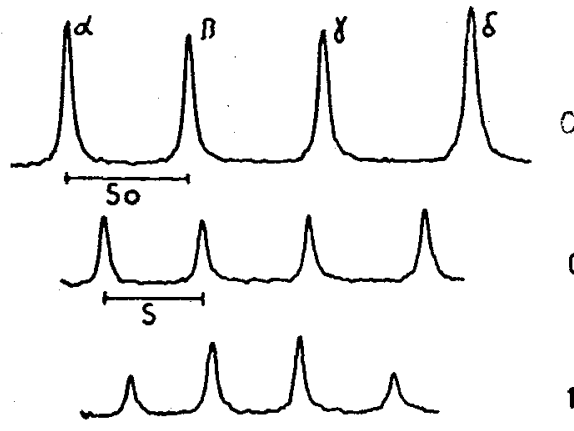

0.91

1.22

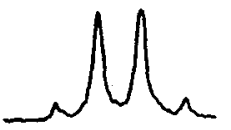

1.82

FIG. 24. - Spectre hyperfin $\left(\Delta m_{9}= \pm 1\right)$ d'atomes $87 \mathrm{Rb}$ habillés par des photons $R F$. Le chiffre situé à droite de chaque courbe repère la valeur correspondante de $\omega_{1} / \omega$. Résultats expérimentaux.

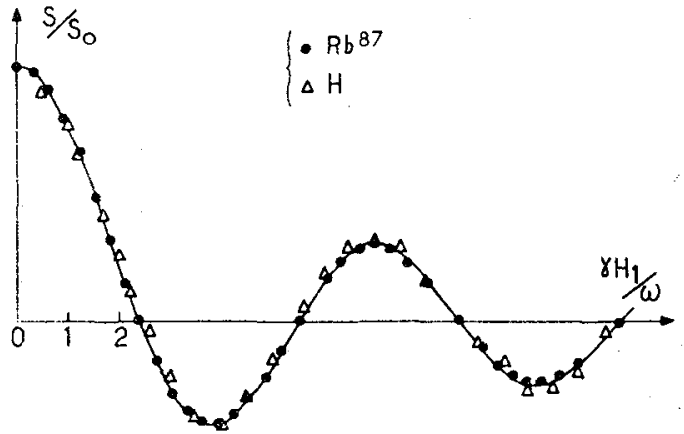

FIG. 25. - Résultats expérimentaux concernant l'écart des raies du spectre hyperfin $\left(\Delta m_{\mathrm{r}}= \pm 1\right)$ d'atomes $H$ et ${ }^{87} \mathrm{Rb}$ habillés par des photons $R F$. s : écart entre les raies d'un atome habillé; $s_{0}$ : écart entre les raies d'un atome libre $\left(\omega_{1}=\gamma H_{1}=0\right)(0:$ résultats concernant ${ }^{87} \mathrm{Rb} ; \boldsymbol{A}$ : résultats concernant $H$ ). La courbe représente la fonction $J_{0}\left(\omega_{1} / \omega\right)$.

des temps de relaxation dans l'état fondamental, due à l'utilisation de cellules dont les parois internes sont recouvertes d'un enduit paraffiné [26]. De plus, le rapport signal sur bruit est excellent (de l'ordre de 3000 ) par suite de la possibilité d'utiliser des techniques d'amplification sélective et de détection synchrone. La figure 27 montre un exemple des courbes expérimentales obtenues. 

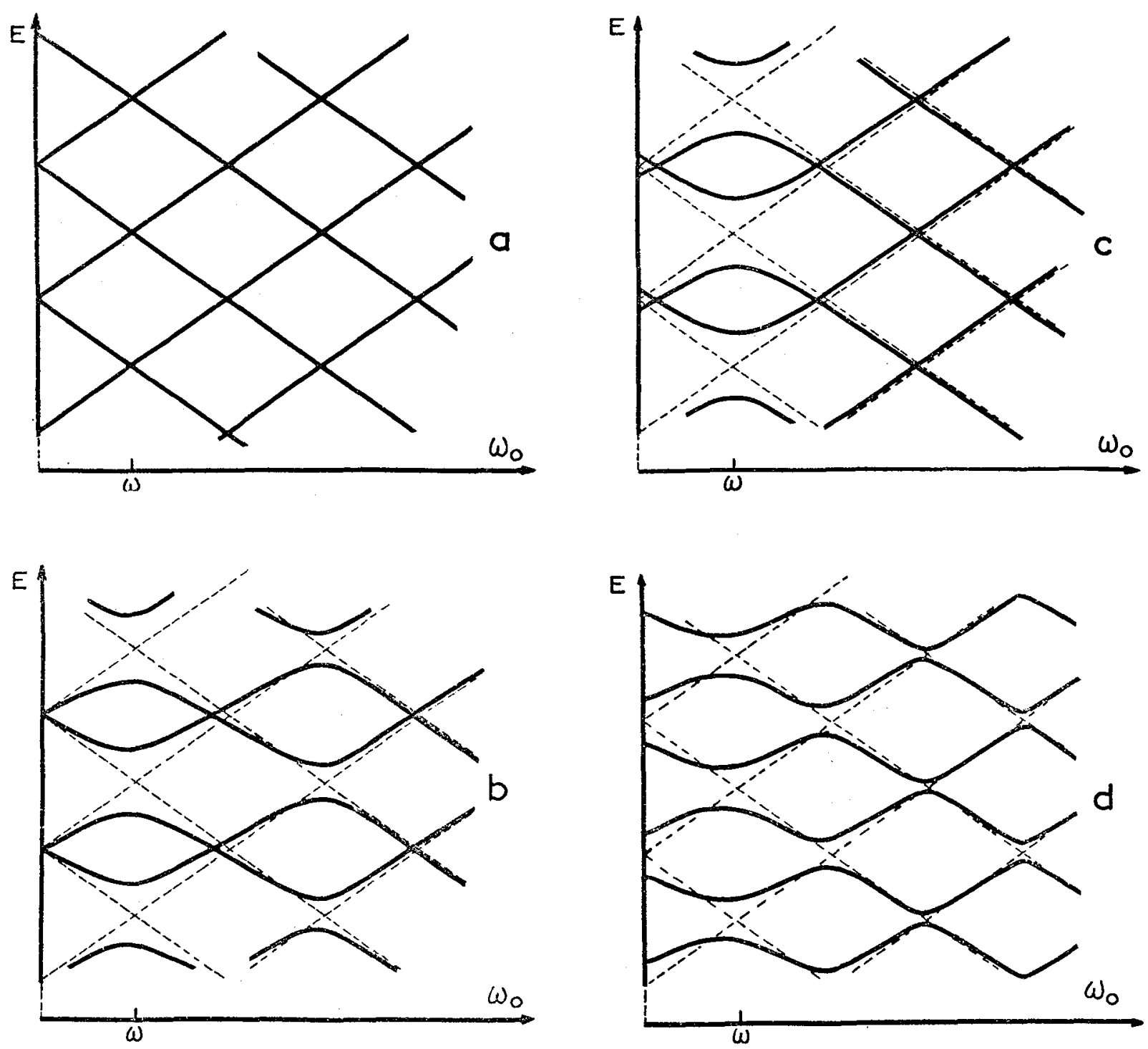

Fig. 26. - Diagrammes Zeeman d'atomes habillés par des champs $R F$ de polarisations diverses. Le moment cinétique de l'atome est $I=\frac{1}{2}, \dot{a}$ : Champ $R F$ de polarisation linéaire parallèle à $\mathrm{H}_{0} ; b:$ Champ $R F$ de polarisation linéaire perpendiculaire à $\mathbf{H}_{0} ; c$ : Champ $R F$ tournant dans un plan perpendiculaire à $\mathbf{H}_{0} ; d:$ Champ $R F$ tournant dans un plan parallele à $\mathbf{H}_{0}$.

Une telle finesse nécessite bien sûr de protéger la cellule de résonance du bruit magnétique nécessairement présent dans le laboratoire. L'expérience est donc réalisée à l'intérieur d'un blindage magnétique de haute qualité ( 5 enceintes concentriques de mumétal de $2 \mathrm{~mm}$ d'épaisseur). La figure 28 donne un schéma général du montage expérimental.

Plaçons-nous au centre de la courbe de résonance de la figure 27, qui a une forme de dispersion, et appliquons à la cellule des variations de champ connues. On peut ainsi tester la sensibilité du dispositif au champ magnétique. La figure 29 montre le signal obtenu lorsqu'on applique des créneaux de champ de $2 \times 10^{-9} \mathrm{G}$. On voit qu'on a réalisé ainsi un magnétomètre très sensible.

Sa sensibilité est suffisante d'ailleurs pour détecter le champ statique créé à une distance macroscopique par des noyaux de ${ }^{3} \mathrm{He}$ orientés en phase gazeuse par pompage optique. La figure 30 représente le schéma de principe de l'expérience : 2 cellules de résonance contenant respectivement des atomes de ${ }^{87} \mathrm{Rb}$ et de ${ }^{3} \mathrm{He}$ sont placés côte à côte. Les atomes de ${ }^{87} \mathrm{Rb}$ sont « habillés » par le champ $R F \mathbf{H}_{1} \cos \omega t$, et l'on détecte la résonance de croisement de niveaux en champ nul au moyen du faisceau de pompage optique transversal $F_{1}$. Les noyaux de ${ }^{3} \mathrm{He}$ sont orientés par pompage optique au moyen du faisceau $\mathrm{F}_{2}$. Ils pointent donc le long de la direction de $\mathrm{F}_{2}$ et le signal obtenu sur $F_{1}$ permet de détecter le champ statique qu'ils créent au centre de la cellule de ${ }^{87} \mathrm{Rb}$ (situé à une distance de $6 \mathrm{~cm}$ ).

$\mathrm{Si}$ après avoir orienté ces noyaux, on les fait tourner 


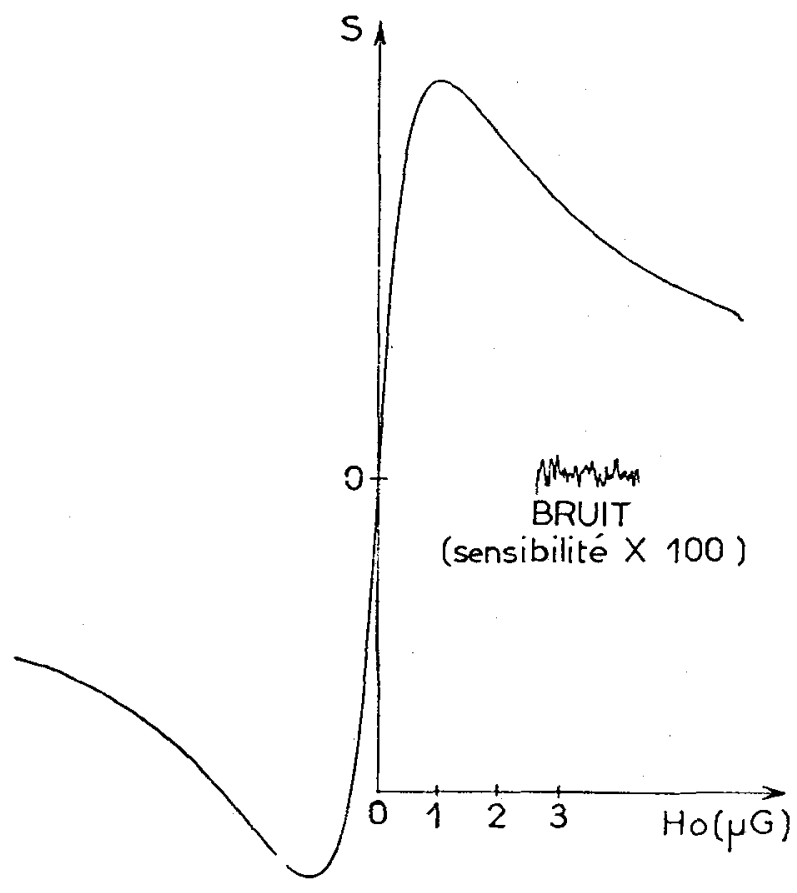

Fig. 27. - Résonance de croisement de niveaux observée autour du champ nul sur des atomes ${ }^{87} \mathrm{Rb}$ habillés par un champ $R F$ de polarisation linéaire parallèle à $\mathbf{H}_{0}$. La détection se fait sur la modulation à la fréquence $\omega / 2 \pi$ des signaux optiques (lumière absorbée). Le bruit apparaît lorsqu'on multiplie la sensibilité par 100.

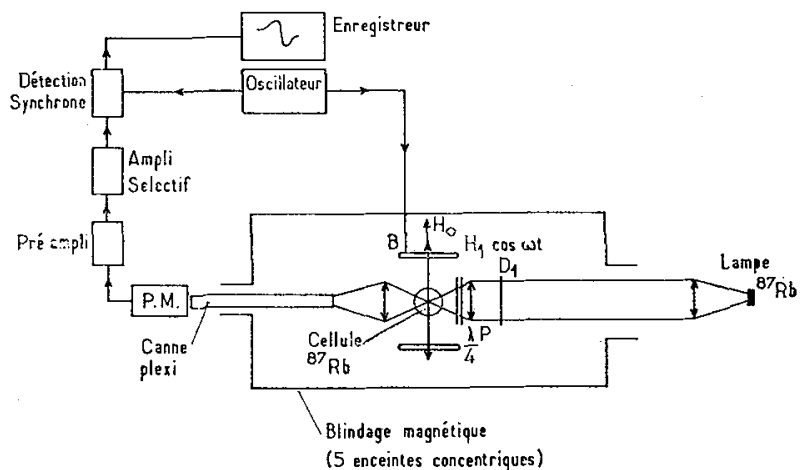

Fig. 28. - Schéma de principe du magnétomètre, $D_{1}:$ filtre laissant passer la raie $D_{1}$ de $87 R b ; P$ : polariseur $\lambda / 4$ : lame quart d'onde ; $\mathbf{B}$ : bobines. L'appareil est sensible à tout champ statique $\mathbf{H}_{0}$ parallèle au champ $R F \mathbf{H}_{1} \cos \omega t$.

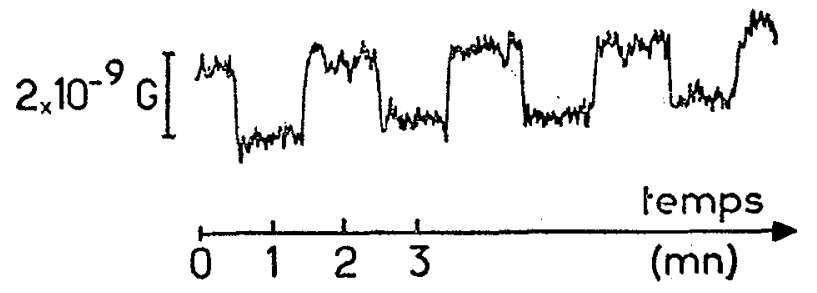

FIG. 29. - Réponse du magnétomètre à des créneaux de champ de $2 \times 10^{-9} \mathrm{G}$. (Constante de temps de la détection synchrone : $3 \mathrm{s.})$ autour d'un champ très faible, appliqué uniquement sur la cellule de ${ }^{3} \mathrm{He}$ perpendiculairement à $\mathrm{F}_{2}$ (période de Larmor de l'ordre de $2 \mathrm{mn}$ ), le champ statique macroscopique qu'ils créent au centre de la cellule

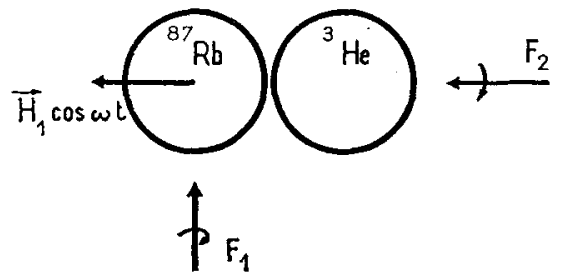

Fig. 30. - Détection magnétostatique du champ magnétique créé à une distance macroscopique par des noyaux de ${ }^{3} \mathrm{He}$ orientés en phase gazeuse par pompage optique. Schéma de principe de l'expérience.

de ${ }^{87} \mathrm{Rb}$ oscille à la même fréquence. La figure 31 montre le signal obtenu.

Au début de la précession, le nombre de noyaux orientés est de l'ordre de $5 \times 10^{15} / \mathrm{cm}^{3}$ dans un volume global de $100 \mathrm{~cm}^{3}$. Le temps de relaxation
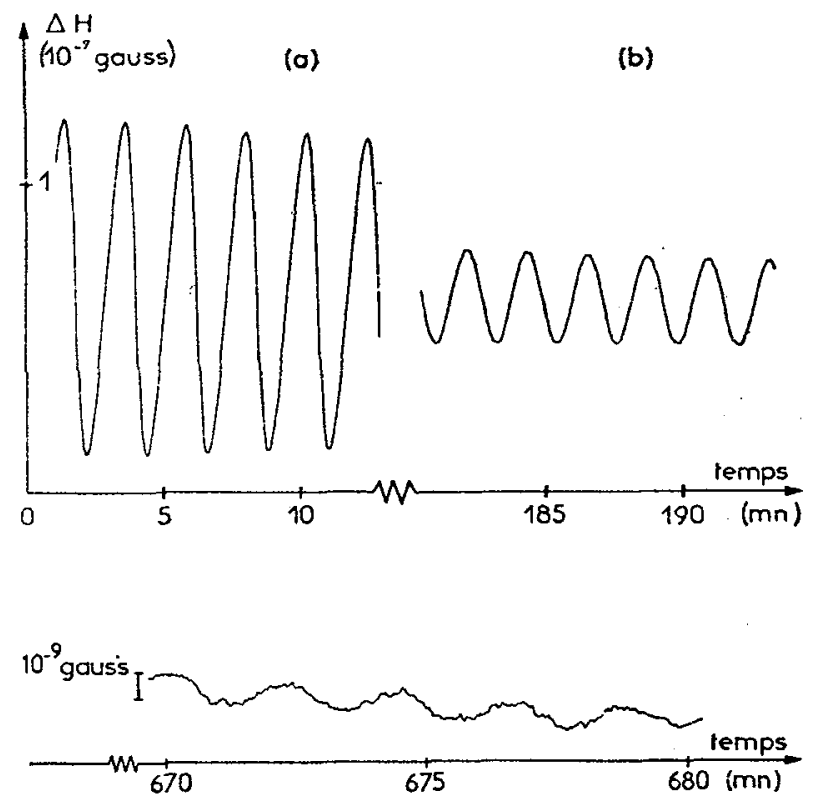

Fig. 31. - Détection magnétostatique de la précession de Larmor de noyaux de ${ }^{3} \mathrm{He}$. Résultats expérimentaux.

de ${ }^{3} \mathrm{He}$ est très long et on peut observer leur précession de Larmor pendant plusieurs heures. Le signal est encore visible au bout de 11 heures et correspond seulement à $5 \times 10^{13}$ noyaux orientés par $\mathrm{cm}^{3}$. On voit que la sensibilité de ce procédé de détection magnétostatique du magnétisme nucléaire est considérable. 


\section{Bibliographie}

[1] Une telle propriété est à la base de la méthode de double résonance.

Brossel (J.), Kastler (A.), C. R. Acad. Sci. Patis, $1949,229,1213$.

Brossel (J.), Bitter (F.), Phys. Rev., 1952, 7, 622.

[2] Kastler (A.), J. Physique Rad., 1950, 11, 255.

[3] Un certain nombre de cours récents relatifs aux effets importants de l'électrodynamique quantique (Lamb-shift, $g-2, \ldots$ ) peuvent être trouvés dans la référence.

Cargese Lectures in Physics, Vol. 2 edited by M. Lévy (Gordon and Breach, 1967).

Cet ouvrage contient également un cours de l'auteur portant sur certains des effets décrits dans le présent article.

[4] Messiah (A.), Mécanique quantique, Tome II, Dunod, 1964.

[5] Barrat (J. P.), Cohen-Tannoudji (C.), J. Physique Rad., 1961, 22, 329 et 443.

[6] Cohen-Tannoudji (C.), Thèse Paris 1962; Ann. de Phys., 1962, 7, 423 et 469.

[7] Aleksandrov (E. B.), Bonch-Bruevich (A. M.), Kostin (N. N.), Khodovor (V. A.) ; Soviet Phys. - JETP Letters, 1966, 3, 53 et Soviet Phys. JETP Letters, 1969, 29, 82.

Bradley (D. J.), Durrant (A. J. F.), Gale (G. M.), Sмттн (P. D.), IEEE, J. Quant. Electr., 1968, QE-4, 707.

Platz (P.), Appl. Phys. Lett., 1969, 14, 198; ibid., 1970, 16,70 .

[8] Dupont-Roc (J.), Polonsky (N.), Cohen-Tannoudj (C.), Kastler (A.), C. R. Acad. Sci. Paris, 1967, 264, 1811.

[9] Dupont-Roc (J.), Polonsky (N.), Cohen-Tannoudji (C.), Kastler (A.), Phys. Letters, 1967, 25A, 87.

[10] Happer (W.), Mathur (B. S.), Phys. Rev., 1967, 163, 12.

[11] Dupont-Roc (J.), Cohen-Tannoudji (C.), C. R. Acad. Sci. Paris, 1968, 267, 1211.

[12] Arditi (M.), Carver (T. R.), Phys. Rev., 1961, 124, 800.

[13] Mathur (B. S.), Tang (H.), Happer (W.), Phys. Rev., 1968, 171, 11.

[14] Conen-Tannoudi (C.), Dupont-Roc (J.), Phys. Rev. A paraître.

[15] Happer (W.), Mathur (B. S.), Phys. Rev. Letters, $1967,18,727$.

[16] Dupont-Roc (J.), Cohen-Tannoudis (C.), C. R. Acad. Sci. Paris, 1968, 267, 1275.

[17] Haroche (S.), Thèse, Paris, 1971, Ann. de Phys. A paraître.
[18] Cohen-Tannoudji (C.), Haroche (S.), C. R. Acad. Sci. Paris, 1966, 262, 268. J. Physique Rad., 1969, 30,125 et 153.

[19] Polonsky (N.), Cohen-Tannoudji (C.), J. Physique Rad., 1965, 26, 409.

[20] LANDrÉ (C.), Thèse $3^{e}$ Cycle Paris, 1970.

Landré (C.), Cohen-Tannoudi (C.), Dupont-Roc (J.), Haroche (S.), J. Physique, 1970, 31, 971.

[21] Haroche (S.), Cohen-Tannoudi (C.), Audoin (C.), SchermanN (J.-P.), Phys. Rev. Letters, 1970, 24, 861.

Une autre application de la possibilité de faire varier $g$ est décrite dans la référence.

Haroche (S.), Cohen-Tannoudir (C.), Phys. Rev. Letters, 1970, 24, 974.

[22] Cohen-Tannoudj (C.), Haroche (S.), C. R. Acad.Sci. Paris, 1966, 262, 37.

Voir aussi l'article de ces 2 auteurs dans : « Polarisation, Matière et Rayonnement ), Livre de Jubilé en l'honneur d'Alfred Kastler, édité par la Société Française de Physique. Presses Universitaires de France (Paris, 1969).

[23] Winter (J. M.), Thèse, Paris (1958), Ann. de Phys., $1959,4,745$.

[24] Pour un champ $R F$ de polarisation linéaire, parallèle $\grave{a} \mathbf{H}_{0}$ :

Alexandrov (E. B.), Constantivov (O. B.), Pereli (B. I.), Khodovoy (B. A.), J. Exp. Theoret. Phys. (U. S. S. R.), 1963, 45, 503.

Favre (C. J.), GeneuX (E.), Phys. letters, 1964, 8, 190.

Polonsky (N.), Cohen-Tannoudji (C.), C. R. Acad. Sci. Paris, 1965, 26, 409.

Pour un champ $R F$ de polarisation linéaire, perpendiculaire à $\mathbf{H}_{0}$ :

Cohen-Tannoudit (C.), Haroche (S.), C. R. Acad. Sci. Paris, 1965, 261, 5400.

Pour un champ $R F$ tournant dans un plan perpendiculaire à $\mathbf{H}_{0}$ :

Dodd (J. N.), Series (G. W.), Proc. Roy. Soc., 1961, A 263, 353.

Dodo (J. N.), Series (G. W.), Taylor (M. J.), Proc. Roy. Soc., 1963, A 273, 41.

[25] Magnétomètre à $87 \mathrm{Rb}$ et applications :

Dupont-Roc (J.), Haroche (S.), Cohen-TannoudiI (C.), Phys. letters, 1969, 28A, 638.

Cohen-Tannoudit (C.), Dupont-Roc (J.), Haroche (S.), Laloe (F.), Phys. Rev. letters, 1969, 22, 758 et Rev. Phys. Appl., 1970, 5, 95 et 102.

Duront-Roc (J.), Revue Phys. Appliquée, 1970, 5, 853 et J. Phys. Rad., 1971, 32, 135.

[26] Bouchat (M. A.), Brossel (J.), Phys. Rev., 1966, 147, 41. 